\title{
Optimal portfolios in the presence of stress scenarios A worst-case approach
}

\author{
Ralf Korn ${ }^{1,2}$ (D) Lukas Müller ${ }^{1}$ D
}

Received: 10 December 2020 / Accepted: 14 July 2021 / Published online: 14 August 2021

(c) The Author(s) 2021

\begin{abstract}
Insurance companies and banks regularly have to face stress tests performed by regulatory instances. To model their investment decision problems that includes stress scenarios, we propose the worst-case portfolio approach. Thus, the resulting optimal portfolios are already stress test prone by construction. A central issue of the worst-case portfolio approach is that neither the time nor the order of occurrence of the stress scenarios are known. Even more, there are no probabilistic assumptions regarding the occurrence of the stresses. By defining the relative worst-case loss and introducing the concept of minimum constant portfolio processes, we generalize the traditional concepts of the indifference frontier and the indifference-optimality principle. We prove the existence of a minimum constant portfolio process that is optimal for the multi-stress worst-case problem. As a main result we derive a verification theorem that provides conditions on Lagrange multipliers and nonlinear ordinary differential equations that support the construction of optimal worst-case portfolio strategies. The practical applicability of the verification theorem is demonstrated via numerical solution of various worst-case problems with stresses. There, it is in particular shown that an investor who chooses the worst-case optimal portfolio process may have a preference regarding the order of stresses, but there may also be stress scenarios where he/she is indifferent regarding the order and time of occurrence.
\end{abstract}

Keywords Optimal portfolios $\cdot$ Stress scenarios $\cdot$ Indifference principle $\cdot$ Minimum constant portfolio $\cdot$ Constrained optimization

JEL classification $\mathrm{G} 01 \cdot \mathrm{G} 11 \cdot \mathrm{G} 21 \cdot \mathrm{G} 22 \cdot \mathrm{G} 32$

Ralf Korn

korn@mathematik.uni-kl.de

Lukas Müller

lmmuelle@mathematik.uni-kl.de

1 Fachbereich Mathematik, TU Kaiserslautern, 67653 Kaiserslautern, Germany

2 Fraunhofer Institute ITWM, Fraunhofer Platz 1, 67663 Kaiserslautern, Germany 


\section{Introduction}

Stress tests for banks and insurance companies performed by regulatory instances such as e.g. the European Central Bank are nowadays even an issue of public news. The usual way how these stresses are considered in finding an optimal investment strategy is to first find a good strategy with respect to a primal goal (such as expected utility). Then, it is checked if the resulting strategy also performs acceptable when stresses occur, possibly has to be modified, and the checking is repeated until all stress criteria are met.

We will in this article present an alternative approach which directly includes the effects of possible stresses in the investment problem. This new approach consists of modifying the so-called worst-case scenario approach to portfolio optimization for explicitly considering the occurrence of stress scenarios. Consequently, the trial-and-error character of the usual approach can be avoided.

Our approach is mainly motivated by regulatory texts. Indeed, in their methodological principles of insurance stress testing [4], the European Insurance and Occupational Pensions Authority (EIOPA) gives the following definition of stress scenarios:

Stress scenarios are severe but plausible hypothetical situations that can adversely affect the balance sheets and solvency positions of insurance undertakings. Scenarios can be compromise a single shock or a combination of market, demographic, financial and insurance-specific shocks that are expected to affect the resilience of individual undertakings and the insurance sector as a whole.

Further, EIOPA [4] states that so-called equity shocks "are provided in terms of percentage changes". A concrete example of a shock is given in the Solvency II Delegated Act Articles 168-173 [3], which deals with the equity risk sub-module of the market risk module of the standard formula. Here equities are divided into Type 1 and Type 2, depending on where they are listed. Apart from symmetrical adjustments and further exceptions, a stress of Type 1 equities is a market decline of 39\%, wheres for Type 2 equities the decline is $49 \%$.

As usual in the worst-case scenario approach introduced by Korn and Wilmott [13], we assume that the stresses can all happen, but make no probabilistic assumption if and when they occur, i.e. with regard to this we follow the concept of Knightian uncertainty (see [7]).

The importance to add stress scenarios to the investment decision problem and not to make any probabilistic assumptions regarding the occurrence of the scenarios is justified by the Insurance Regulation Committee of the International Actuarial Association [6]:

The likelihood and financial effect of certain scenarios can be extremely uncertain. In these cases it is almost impossible to precisely estimate their small probabilities. In fact, their effect cannot be estimated and even their identification cannot be easily made through the application of a traditional economic capital model.... Yet, such scenarios may lead to the largest financial strain for firms or an entire industry. The use of scenario analysis and stress testing by decision makers and regulators may prove to be the best approach to prioritize their options, whether to add to capital or to use other approaches to mitigate these risks.

Solving the worst-case scenario optimal portfolio approach, introduced by Korn and Wilmott in [13] in the case of the logarithmic utility function, has been extended in [8] and [10] by using the indifference principle. In [12] the worst-case portfolio problem is embedded in a classical HJB-framework and a connection to stochastic control theory is established. Seifried introduced two concepts in [20], which are also of great importance for 
this work, namely the indifference frontier and the indifference optimality principle (see also [11]), to generalize the results to more general utility functions and price dynamics.

In the course of this work, we will see in particular that the portfolio optimization problems considered in [12] and [9], among others, are in fact special cases of the problem introduced here. More precisely, we have generalised the problem with one stock and $n \geq 1$ identical stresses stated in [12]. Also, our model includes the multi-asset problem with one stress in [9] as a simple special case.

We will present details of the worst-case scenario approach in the next section, but will already point out that conventional portfolio optimization methods-such as the stochastic control approach introduced by Merton [15] or the martingale approach as introduced (among others) by Pliska [18] — are not applicable as we have neither information on the jump times nor the jump intensity.

The latter fact distinguishes the worst-case approach from the so-called ambiguity approaches which are also worst-case approaches to portfolio optimization, but typically focus on the parameters of the market, e.g., stock price coefficients or the underlying probability measure. Examples of this approach are e.g. Talay and Zheng [21] where the market acts against the interest of the trader and chooses the market coefficients or Pflug and Wozabal [17] who take into account the ambiguity in choosing the probability model, i.e. the underlying probability model is not perfectly known. In [19], Schied considers a set of probability measures to maximize the robust utility of the terminal wealth in a complete market model and in [5] the standard mean-variance model is extended to model ambiguity aversion via a minimization over the priors. A generalization of the classical portfolio and consumption model of an ambiguity type that can be solved by a generalized HJB-equation is given in Lin and Riedel [14] and in [1], Biagini and Pinar consider a robust Merton problem using a max-min Hamilton-Jacobi-Bellman-Isaacs PDE. Also in these ambiguity approaches, there is the possibility of integrating uncertainty with regard to jump processes. Neufeld and Nutz [16] study a robust portfolio optimization problem with uncertain drift, volatility, and jump characteristics of the risky assets dynamics by defining a set of possible Lévy triplets. However, in such a model, the investor is only hedged in the mean against a jump.

A key challenge in our model is that the investor is not only faced with the challenge of when or if a stress occurs, rather that there are different types of stresses. Therefore there is the additional uncertainty of not knowing in which order the stresses occur. Due to this additional uncertainty component the existing results cannot simply be adapted, which is why in this work we have to introduce new concepts and have to derive new results such as

- the extension of the worst-case concept to different types of stresses,

- allowing for an unknown sequential occurrence of the different types of stresses,

- the concept of minimum constant portfolio processes,

- the proof of existence of an optimal minimum constant portfolio process,

- a verification theorem for the resulting multi-stress-type multi-asset worst-case problem.

While our definitions and the setting will be given for the general case of $n \geq 1$ stresses, we will in this work mostly focus on the case of $n=2$ stresses. The extension to the general case of $n \geq 2$ is described in Remark 7 .

We start with the introduction of the the worst-case concept in Sect. 2. Then, in Sects. 3 and 4, we solve the portfolio optimization problem in the stress-free world and the problem when only one stress is left. In Sect. 5, we derive the minimum constant principle and solve the multi-asset multi-stress worst-case problem. The preceding considerations then enable us to formulate a verification theorem in Sect. 6. The heuristic construction of the optimal strategy and numerical examples are shown in Sect. 7. 


\section{Worst-case portfolio optimization}

In this section, we introduce the mathematical framework that allows us to study a portfolio optimization problem in a financial market with stresses.

Basic mathematical assumptions. Throughout this work we fix a time horizon $T>0$. All processes are defined on the complete probability space $(\Omega, \mathcal{F}, P)$.

Asset price dynamics and stress scenarios. We consider a capital market with a money market account with dynamic evolution given by

$$
d B(t)=B(t) r d t, \quad B(0)=1,
$$

and $d$ stocks with prices $S_{i}(t), i=1, \ldots, d$. Before introducing the stock price dynamics and the suitable filtration on this probability space, we need to introduce the notion of a stress scenario.

Definition 1 A vector $z \in \mathbb{R}^{d}$ with components $z_{i} \in[0,1)$ is called a stress. A pair $(z, \xi)$, consisting of a stress $z$ and a stochastic process $\xi$ with values in $[0, T] \cup\{\infty\}$, is called a stress scenario. We will refer to $\xi$ as the time of occurrence of the stress $z$, where we formally set $\{\xi=\infty\}$ if the stress does not occur. For a stress scenario $(z, \xi)$, we further introduce the (indicator) jump process

$$
J_{z}(t):=\mathbb{1}_{\{\xi \leq t\}}, t \in[0, T] .
$$

Let $W(t)$ be a $d$-dimensional Brownian motion and consider $n$ stress scenarios $\left(z^{(1)}, \xi_{1}\right), \ldots,\left(z^{(n)}, \xi_{n}\right)$ with corresponding jump processes $J_{j}(t):=J_{z^{(j)}}(t), j=1, \ldots, n$. We then assume that our probability space is equipped with a filtration $\mathbb{F}=\left(\mathcal{F}_{t}\right)_{t \in[0, T]}$ that satisfies the so-called usual conditions and that includes both, the filtration generated by the Brownian motion $W(t)$ and the one generated by the jump processes $J_{1}(t), \ldots, J_{n}(t)$. To be able to include the scenario $\left\{\xi_{j}=\infty\right\}$, for $j \in\{1, \ldots, n\}$, we extend $\mathbb{F}$ to $[0, T] \cup\{\infty\}$ by letting $\mathcal{F}_{\infty}:=\mathcal{F}_{T}$. Note in particular that then all $\xi_{j}$ are stopping times with respect to this filtratrion. We denote this by $\xi_{j} \in \Theta$, i.e. $\Theta$ is defined as the set of $[0, T] \cup\{\infty\}$-valued stopping times.

We are now ready to define the stock price dynamics, for $i=1, \ldots, d$, via the following jump diffusion process

$$
d S_{i}(t)=S_{i}(t-)\left(b_{i} d t+\sum_{j=1}^{d} \sigma_{i j} d W_{j}(t)-\sum_{j=1}^{n} z_{i}^{(j)} d J_{j}(t)\right), \quad S_{i}(0)=s_{0, i} .
$$

with the drift vector $b=\left(b_{1}, \ldots, b_{d}\right)^{T}$ and the volatility matrix $\sigma=\left(\sigma_{i j}\right)$ of full rank.

Our interpretation of Knightian uncertainty about the occurrence of the stresses is, that we can of course observe the jump processes $J_{j}(t)$ for $j \in\{1, \ldots, n\}$, but have no knowledge about the likelihood of the actual appearance of the jumps. In particular, we have also no knowledge on the dynamic properties of the jump processes, such as their possible intensities.

To be in line with our motivating example of stress tests, we assume that each stress can occur at most once on $[0, T]$.

Portfolio strategies. Let us consider an investor with an initial capital $x>0$, which he/she can invest in $d$ stocks and the money market account. Furthermore, he/she can observe a stress scenario and react accordingly after its occurrence. We describe the investor's behaviour by a self-financing portfolio process $\pi$ which denotes the fractions of his/her wealth invested in the stocks. We assume $\pi$ to be predictable to model that a reallocation of holdings is not 
possible during the occurrence of a stress. However, it should also avoid a ruin by using a cautious investment strategy. Technically, we thus define the following set of admissible portfolio processes.

Definition 2 Let $z^{(1)}, \ldots, z^{(n)}$ be $n$ stresses with corresponding times of occurrence $\xi_{1}, \ldots, \xi_{n} \in \theta$. Then, the set of admissible portfolio processes $\pi$ corresponding to an initial capital of $x>0$ at time $t \in[0, T], \mathbb{A}(t, x)$, consists of $\left\{\mathcal{F}_{s}, t \leq s \leq T\right\}$-predictable processes $\pi$ such that

1. the investor's wealth process $X^{\pi}$ is given as the unique solution of the stochastic differential equation

$$
\begin{aligned}
X^{\pi}(t) & =x, \\
d X^{\pi}(s) & =X^{\pi}(s-)\left(\left(r+\pi(s)^{T}(b-r \underline{1})\right) d s+\pi(s)^{T} \sigma d W(s)\right. \\
& \left.-\sum_{j=1}^{n} \pi(s)^{T} z^{(j)} d J_{j}(s)\right),
\end{aligned}
$$

for $s \in[t, T]$.

2. $\pi(s)$, for $s \in[t, T]$, is of the form

$$
\pi(s)=\sum_{u \in\{0,1\}^{n}} \mathbb{1}_{\{\bar{u}(s)=u\}} \pi_{u}(s),
$$

where all $\pi_{u}(s)$ are assumed to be predictable and to have right-continuous paths with left limits on $[0, T]$, for all $u=\left(u_{1}, \ldots, u_{n}\right) \in\{0,1\}^{n}$, and $\bar{u}(s)=\left(\bar{u}_{1}(s), \ldots, \bar{u}_{n}(s)\right) \in$ $\{0,1\}^{n}$, for $s \in[0, T]$, with $\bar{u}_{i}(s)=1$ iff $s \leq \xi_{i}$, for $i \in\{1, \ldots, n\}$.

3. We further require

$$
\begin{gathered}
X^{\pi}(s)>0 \forall s \in[t, T], \\
\int_{t}^{T} \pi_{u, i}^{2}(s) d s<\infty P \text {-a.s. for } i=1, \ldots, d, \text { and } u \in\{0,1\}^{n} .
\end{gathered}
$$

We introduce the abbreviation $\mathbb{A}(x):=\mathbb{A}(0, x)$.

We would like to use the following remark to draw some conclusions from the above definition and to introduce a new notation.

Remark 1 1. If, for $j \in\{1, \ldots, n\}$, a stress $z^{(j)}$ occurs at a time $\xi_{j} \in \Theta, \xi_{j}<\infty$, the wealth process $X^{\pi}$ changes at time $\xi_{j}$ according to

$$
X^{\pi}\left(\xi_{j}\right)=\left(1-\pi\left(\xi_{j}\right)^{T} z^{(j)}\right) X^{\pi}\left(\xi_{j}-\right) .
$$

Note that our definition of the portfolio process ensures that a change to the appropriate $\pi_{u}$ (.) sub strategy follows directly after the jump.

2. The non-negativity condition in particular implies that an investor does not suffer a total loss due to any stress, i.e. for $n$ remaining stresses $z^{(1)}, \ldots, z^{(n)}$ an admissible portfolio process $\pi$ needs to fulfil the condition

$$
\max \left\{\pi(t)^{T} z^{(j)} \mid j=1, \ldots, n\right\}<1,
$$

for $t \in[0, T]$. 
3. In the case of $n$ stress scenarios, we will often denote the wealth process $X^{\pi}$ before the first stress has occurred by $X^{\pi_{(1, \ldots, 1)}}$. This will be of particular interest as we will solve the worst-case problem by building up our solution recursively, starting from the well-known no crash optimal portfolio process.

The worst-case optimization problem. Let $\left(z^{(1)}, \xi_{1}\right), \ldots,\left(z^{(n)}, \xi_{n}\right)$ be $n$ stress scenarios and let $U:(0, \infty) \rightarrow \mathbb{R}$ be the investor's utility function, which is assumed to be strictly concave, increasing and continuously differentiable. We call the problem

$$
\sup _{\pi \in \mathbb{A}(x)} \inf _{\xi_{1}, \ldots, \xi_{n} \in \Theta} \mathbb{E}_{0, x,\left(u_{1}, \ldots, u_{n}\right)}\left(U\left(X^{\pi}(T)\right)\right), u_{1}=\ldots=u_{n}=1,
$$

the worst-case portfolio problem under stresses, where $\mathbb{E}_{t, x,\left(u_{1}, \ldots, u_{n}\right)}$ for $t \in[0, T]$ denotes the conditional expectation given that $X^{\pi}(t)=x$ and $u_{j} \in\{0,1\}, j=1, \ldots, n$, with $u_{j}=1$ if and only if $\xi_{j} \geq t$. These indices are omitted if $t=0$ or if $u_{1}=\ldots=u_{n}=0$.

In the further course of this work, we will look at the case of $d$ stocks and two stresses $z^{(1)}$ and $z^{(2)}$ with corresponding times of occurrence $\xi_{1}, \xi_{2} \in \Theta$, since the limitation to two stress scenarios allows for a simpler notation. However, in Remark 7 we will indicate how the results of this work can be extended to the general problem with $n$ stress scenarios.

Further, we model the investor's preferences by a CRRA (constant relative risk aversion) utility function, i.e.

$$
U(x)=\frac{1}{1-\gamma} x^{1-\gamma} \text { for } \gamma>0, \gamma \neq 1
$$

In Remark 8 and Remark 9 we explain that the main results can be adapted for the case of a logarithmic utility function $U(x)=\ln (x)$, which can be interpreted as the limit case of $\gamma \rightarrow 1$.

We make the additional assumption that only one stress can occur at one time instant. In the following remark we motivate this by a simple example.

Remark 2 We consider the two-stock portfolio process $\pi(t)^{T}=(0.25,0.25)$ and assume that there exist two stresses $z^{(1)}$ and $z^{(2)}$ with

$$
z^{(1)}=(0.5,0)^{T} \text { and } z^{(2)}=(0,0.5)^{T} .
$$

A simultaneous occurrence of the two stresses at time $t$ would intuitively reduce the wealth by $25 \%$. On the other hand we have

$$
1-\left(\left(1-\pi(t)^{T} z^{(2)}\right)\left(1-\pi(t)^{T} z^{(1)}\right)\right)=1-(1-0.5 \cdot 0.25)^{2}=23.44 \% .
$$

Instead, let us now consider the situation in which the two stresses directly follow each other:

$$
1-\left(\left(1-\pi(t-)^{T} z^{(2)}\right)\left(1-\pi(t)^{T} z^{(1)}\right)\right) \text { with } \pi(t-)^{T}=(0.25,0.25) .
$$

Assuming that no trading takes place after the first stress, the following applies: $\pi(t)^{T}=$ $(2 / 7,1 / 7)$, and therefore:

$$
1-\left(\left(1-\pi(t-)^{T} z^{(2)}\right)\left(1-\pi(t)^{T} z^{(1)}\right)\right)=25 \% .
$$

Hence, in order to reflect an intuitive understanding of stresses in our model, we must assume that not more than one stress occurs at one time instant. 


\section{Terminal utility decomposition and the post stress problem}

In this section we consider the optimal post stress strategy, i. e. the optimal strategy after the last possible stress has occurred. For this, we define $\xi_{m}:=\max \left\{\xi_{1}, \xi_{2}\right\}$. As we are here only interested in the optimal strategy after the last stress, we consider the case $\xi_{m}<\infty$. For the sake of simplicity, we will refer to the portfolio process $\pi_{(0,0)}$ as $\pi_{(0)}$, for $\pi \in \mathbb{A}(x)$. Thus, we obtain the post stress problem

$$
\max _{\pi \in \mathbb{A}\left(\xi_{m}, x\right)} \mathbb{E}_{\xi_{m}, x}\left(U\left(X^{\pi}(T)\right)\right),
$$

which is the classical Merton problem with random initial time $\xi_{m}$.

Decomposition of the terminal utility. To solve the post stress problem we first look at a decomposition of the terminal utility. This enables us to solve the Merton problem with random initial time as well as the classical Merton problem.

Proposition 1 (Terminal utility decomposition) Let $z^{(1)}$ and $z^{(2)}$ be two stresses with corresponding times of occurrences $\xi_{1}, \xi_{2}<\infty$. For any admissible portfolio process $\pi \in \mathbb{A}(x)$ we get the following decomposition:

$$
U\left(X^{\pi}(T)\right)=U\left(X^{\pi}\left(\xi_{m}\right)\right) \exp \left((1-\gamma) \int_{\xi_{m}}^{T} \phi_{\gamma}(\pi(t)) d t\right) Y_{T}^{\gamma}(\pi),
$$

where the mapping $\phi_{\gamma}$ is given by

$$
\phi_{\gamma}(\pi)=r+\pi^{T}(b-r \underline{1})-\frac{1}{2} \gamma \pi^{T} \sigma \sigma^{T} \pi
$$

and $Y^{\gamma}(\pi)=\left(Y_{t}^{\gamma}(\pi)\right)_{t \in\left[\xi_{m}, T\right]}$ is a positive $\mathcal{F}_{t}$-martingale with $Y_{\xi_{m}}^{\gamma}(\pi)=1$.

Proof The proof is analogous to the one of Theorem 5 (Change-of-Measure Device) in [11].ם

We obtain an analogue decomposition for the case that no stress occurs, i.e. if $\xi_{1}=\xi_{2}=\{\infty\}$. This decomposition becomes important in Sect. 5, when we have to consider the no stress scenario while looking at the multi-stress worst-case problem.

The stress-free world. In the stress-free post stress world we now have to solve the Merton problem with random initial time. With the help of the above decomposition of the terminal utility we prove that this problem can be solved by maximizing $\phi_{\gamma}$ from the utility decomposition, i.e. a portfolio process $\bar{\pi} \in \mathbb{A}\left(\xi_{m}, x\right)$ is optimal for the post stress problem (1) if $\bar{\pi}_{(0)}=\pi^{*}$, where

$$
\pi^{*}=\arg \max _{\pi \in \mathbb{R}^{d}} \phi_{\gamma}(\pi) .
$$

One can see that the optimal strategy $\pi^{*}$ is constant and independent of $\xi_{m}$ and $x$.

Proposition 2 (Solution of the post stress portfolio problem) For $\phi_{\gamma}$ as in Proposition 1, the optimal strategy $\pi^{*}$ and the corresponding value function $v\left(\xi_{m} ; x\right)$ in the post stress problem (1) with random initial time $\xi_{m} \in \Theta, \xi_{m}<\infty$, are given by

$$
\begin{aligned}
\pi^{*} & =\frac{1}{\gamma}\left(\sigma \sigma^{T}\right)^{-1}(b-r \underline{1}), \text { with } \\
v\left(\xi_{m} ; x\right): & =\max _{\pi \in \mathbb{A}\left(\xi_{m}, x\right)} \mathbb{E}_{\xi_{m}, x}\left(U\left(X^{\pi}(T)\right)\right) \\
& =\frac{x^{1-\gamma}}{1-\gamma} \exp \left((1-\gamma) \int_{\xi_{m}}^{T} \phi^{\gamma}\left(\pi^{*}\right) d s\right) .
\end{aligned}
$$


Proof See [11].

For the interpretation of a stress as a threat we require from now on

$$
0<\left(\pi^{*}\right)^{T} z
$$

for every stress $z$, i.e. the optimal portfolio in the stress-free world should suffer a loss due to a stress $z$.

\section{The one-stress worst-case problem}

We want to approach the multi-stress scenario step wise. Therefore, we first have a look at the one-stress worst-case problem in the multi asset setting:

$$
\sup _{\pi \in \mathbb{A}(x)} \inf _{\xi \in \Theta} \mathbb{E}_{0, x,(1)}\left(U\left(X^{\pi}(T)\right)\right),
$$

where we use the notation $(z, \xi)$ instead of $\left(z^{(1)}, \xi_{1}\right)$. As this problem was discussed in detail in [9], we only recall ideas and results, and introduce essential concepts.

Based on the results of Sect. 3, we assume in this section, without loss of generality, that $\pi_{(0)}(t)=\pi^{*}, t \in[0, T]$, for all $\pi \in \mathbb{A}(x)$, with $\pi^{*}$ defined as in Proposition 2 .

Indifference-optimality and indifference frontier. The following two fundamental concepts for solving worst-case portfolio optimization problems introduced in [20] allow to solve these problems in a very general setting. A central element is the definition of so-called indifference strategies.

Definition 3 We consider a worst-case portfolio problem with a single stress scenario $(z, \xi)$. Let further $v_{0}(t ; x)$ denote the value function for the corresponding optimal portfolio problem in the post stress setting. If there exists an admissible portfolio process $\pi \in \mathbb{A}(x)$ such that the process

$$
v_{0}\left(t ;\left(1-\pi_{(1)}(t)^{T} z\right) X^{\pi_{(1)}}(t-)\right), \quad t \in[0, T] \cup\{\infty\}
$$

is a martingale on $[0, T] \cup\{\infty\}$ for

$$
v_{0}\left(\infty ;\left(1-\pi_{(1)}(\infty)^{T} z\right) X^{\pi_{(1)}}(\infty-)\right):=U\left(X^{\pi_{(1)}}(T)\right),
$$

we call $\pi$ an indifference strategy for the investor.

This definition has two useful properties. First, it is independent of the concrete form of the utility function. Further, the martingale characterization of an indifference strategy makes its optimality proof independent of the actual market setting. We cite the indifference-optimality principle from [11].

Proposition 3 (Indifference-optimality principle) If $\bar{\pi}$ is an indifference strategy, and for all $\pi \in \mathbb{A}(x)$ there exists at least a single time of stress occurrence $\tilde{\xi} \in \Theta$ with

$$
\begin{aligned}
& \mathbb{E}\left(v_{0}\left(t ;\left(1-\pi_{(1)}(\tilde{\xi})^{T} z\right) X^{\pi_{(1)}}(\tilde{\xi}-)\right)\right) \\
\leq & \mathbb{E}\left(v_{0}\left(t ;\left(1-\bar{\pi}_{(1)}(\tilde{\xi})^{T} z\right) X^{\bar{\pi}_{(1)}}(\tilde{\xi}-)\right)\right)
\end{aligned}
$$

then $\bar{\pi}$ is a worst-case optimal portfolio process. 
In Sect. 5, we prove the minimum constant-optimality principle for the multi-stress worst-case problem, which is also based on the idea of worst-case indifference.

From Proposition 2 we know that the value function $v_{0}(t ; x)$ is continuously differentiable with respect to $t$ and twice continuously differentiable in $x$. Thus, application of Itô's rule to $v_{0}\left(t ;\left(1-\pi_{(1)}(t)^{T} z\right) X^{\pi_{(1)}}(t-)\right)$ and setting the resulting integrand in the finite variation part equal to zero, allows to compute an indifference strategy. We will use similar arguments in the proof of Proposition 5.

Now we can introduce the second essential concept, the indifference frontier. For a proof of the following multi-asset statement we refer to [9].

Proposition 4 (Indifference frontier) Let Assumption (2) be valid and let $\pi \in \mathbb{A}(x)$ be an admissible portfolio process for the one-stress worst-case portfolio problem with stress scenario $(z, \xi)$. Further, let $\bar{\pi}$ be an indifference strategy. Set

$$
\tau:=\inf \left\{t: \pi_{(1)}(t)^{T} z>\bar{\pi}_{(1)}(t)^{T} z\right\}
$$

and define

$$
\tilde{\pi}_{(1)}(t):=\pi_{(1)}(t) \text { if } t<\tau \text { and } \tilde{\pi}_{(1)}(t):=\bar{\pi}_{(1)}(t) \text { if } t \geq \tau \text {. }
$$

Then, we have $\tilde{\pi} \in \mathbb{A}(x)$ and the worst-case bound attained by $\tilde{\pi}$ is at least as big as that achieved by $\pi$.

Hence, only a portfolio process which does not exceed the indifference frontier of an indifference strategy $\bar{\pi}$, i.e. which does not exceed the indifference level $\bar{\pi}_{(1)}(t)^{T} z$ for $t \in[0, T]$, can be a worst-case optimal portfolio process.

Solution of the one-stress worst-case problem. In the multi-asset scenario we lose the uniqueness of the indifference strategy of the single stock case. This is overcome in [9] by using Lagrangian-multiplier methods to figure out the worst-case optimal indifference strategy.

Theorem 1 (Solution of the one-stress worst-case problem) Let Assumption (2) be satisfied and assume a single stress scenario $(z, \xi)$. The optimal portfolio process is the indifference strategy $\bar{\pi}$, given by

$$
\bar{\pi}_{(1)}(t)=\pi^{*}-\frac{z^{T} \pi^{*}-N(t)}{z^{T}\left(\sigma \sigma^{T}\right)^{-1} z}\left(\sigma \sigma^{T}\right)^{-1} z,
$$

where $N(t)$ is the unique solution of the $O D E$

$$
N^{\prime}(t)=-\frac{1}{2 z^{T}\left(\sigma \sigma^{T}\right)^{-1} z} \gamma(1-N(t))\left(z^{T} \pi^{*}-N(t)\right)^{2}
$$

with final condition $N(T)=0$. The corresponding value function $v_{1}(t ; x)$ is

$$
\begin{aligned}
v_{1}(t ; x) & :=\sup _{\pi \in \mathbb{A}(t, x)} \inf _{\xi \in \Theta_{t}} \mathbb{E}_{t, x,(1)}\left(U\left(X^{\pi}(T)\right)\right)=v_{0}\left(t ;\left(1-\bar{\pi}_{(1)}(t)^{T} z\right) x\right) \\
& =\mathbb{E}_{t, x}\left(U\left(X^{\bar{\pi}_{(1)}}(T)\right)\right)
\end{aligned}
$$

for $t \in[0, T]$, where $\Theta_{t}$ denotes the class of $[t, T] \cup\{\infty\}$-valued stopping times.

Proof See [9].

We would like to prove some more properties of the relative wealth loss $\bar{\pi}_{(1)}^{T} z$ of the optimal strategy in case that at most one stress can occur. 
Lemma 1 (Decreasing wealth loss) Assume a single stress scenario $(z, \xi)$ and let $\bar{\pi}$ be given as in Theorem 1. Then we obtain

$$
0 \leq \bar{\pi}_{(1)}(t)^{T} z<\min \left\{\left(\pi^{*}\right)^{T} z, 1\right\}
$$

and thus in particular $\left(\bar{\pi}_{(1)}^{T} z\right)^{\prime}(t)<0$ and $\left(\bar{\pi}_{(1)}^{T} z\right)^{\prime \prime}(t)<0$ for all $t \in[0, T]$.

Proof Note that for $N$ defined in Theorem 1, we obtain $N(t)=\bar{\pi}_{(1)}(t)^{T} z$ for all $t \in[0, T]$. Therefore it is enough to show that $N$ fulfills the above properties. The inequalities $0 \leq$ $N(t)<1$ for all $t \in[0, T]$ hold by Lemma 4.2. in [20]. We now use similar arguments to prove $N(t)<\left(\pi^{*}\right)^{T} z$ for all $t \in[0, T]$. Equation (3) implies for $t \in[0, T]$ :

$$
\frac{\partial}{\partial t} \log \left(z^{T} \pi^{*}-N(t)\right)=\frac{1}{2 z^{T}\left(\sigma \sigma^{T}\right)^{-1} z} \gamma(1-N(t))\left(z^{T} \pi^{*}-N(t)\right)
$$

if $z^{T} \pi^{*}>N(t)$. Integrating both sides, applying the exponential function and using $N(T)=$ 0 leads to

$$
\frac{z^{T} \pi^{*}}{z^{T} \pi^{*}-N(t)}=\exp \left\{\int_{t}^{T} \frac{1}{2 z^{T}\left(\sigma \sigma^{T}\right)^{-1} z} \gamma(1-N(s))\left(z^{T} \pi^{*}-N(s)\right) d s\right\}
$$

for $t \in[0, T]$, if $z^{T} \pi^{*}>N(s)$ for $s \in[t, T]$. The above integrand is a continuous function in $N$ and $N$ is continuous itself. Therefore there exists an upper bound $M>0$ such that for $t \in[0, T]$

$$
\exp \left\{\int_{t}^{T} \frac{1}{2 z^{T}\left(\sigma \sigma^{T}\right)^{-1} z} \gamma(1-N(s))\left(z^{T} \pi^{*}-N(s)\right) d s\right\} \leq \exp \{M(T-t)\},
$$

and therefore

$$
z^{T} \pi^{*}-N(t) \geq \exp \{-M(T-t)\} z^{T} \pi^{*},
$$

if $z^{T} \pi^{*}>N(s)$ for $s \in[t, T]$. We define $\delta=\frac{1}{2} \exp \{-M T\} z^{T} \pi^{*}$ and since, by assumption (2), $z^{T} \pi^{*}>0$ we get $\delta>0$. Then we obtain for $t \in[0, T]$ that

$$
z^{T} \pi^{*}-N(t) \geq 2 \delta
$$

if $z^{T} \pi^{*}>N(s)$ for $s \in[t, T]$. Now we define $\tilde{t}$ as

$$
\tilde{t}:=\inf \left\{t \in[0, T] \mid z^{T} \pi^{*}-N(s) \geq \delta \text { for all } s \in[t, T]\right\} .
$$

The infimum is attained, since $z^{T} \pi^{*}-N(T)=\exp \{M T\} 2 \delta \geq \delta$. Now we prove by contradiction that $\tilde{t}=0$. For this, assume $\tilde{t}>0$. Inequality (4) and the definition of $\tilde{t}$ lead to $z^{T} \pi^{*}-N(\tilde{t}) \geq 2 \delta$. By continuity, there exists $\bar{t}<\tilde{t}$, with $z^{T} \pi^{*}-N(s) \geq \delta$ for all $s \in[\bar{t}, T]$, which is a contradiction to the definition of $\tilde{t}$. Therefore we get $\tilde{t}=0$ and $N(t)<\left(\pi^{*}\right)^{T} z$ for all $t \in[0, T]$.

With $\bar{\pi}_{(1)}(t)^{T} z<\min \left\{\left(\pi^{*}\right)^{T} z, 1\right\}$ and Eq. (3) we directly get $N^{\prime}(t)=\left(\bar{\pi}_{(1)}^{T} z\right)^{\prime}(t)<0$ for all $t \in[0, T]$. The second derivative of $N$ is for $t \in[0, T]$ given by

$$
N^{\prime \prime}(t)=K N^{\prime}(t)\left(z^{T} \pi^{*}-N(t)\right)\left(2(1-N(t))+\left(z^{T} \pi^{*}-N(t)\right)\right),
$$

for a constant $K>0$. With the above considerations it follows that $N^{\prime \prime}(t)=\left(\bar{\pi}_{(1)}^{T} z\right)^{\prime \prime}(t)<0$ for all $t \in[0, T]$. 
Note that $\bar{\pi}$ is admissible in terms of Definition 2 and the optimal strategy is independent of the wealth $x$. Further, the optimal sub strategy $\bar{\pi}_{(1)}$ from Theorem 1 is deterministic. Therefore, even in the multi-stress worst-case scenario we can assume that we know the optimal portfolio process when only one stress can still occur.

\section{The multi-stress worst-case problem}

Now we are able to consider the worst-case portfolio problem under stresses with two stress scenarios $\left(z^{(1)}, \xi_{1}\right)$ and $\left(z^{(2)}, \xi_{2}\right)$. Hence, we look at the following portfolio optimization problem:

$$
\sup _{\pi \in \mathbb{A}(x)} \inf _{\substack{\xi_{1}, \xi_{2} \in \Theta \\ \xi_{1} \neq \xi_{2}}} \mathbb{E}_{0, x,(1,1)}\left(U\left(X^{\pi}(T)\right)\right),
$$

where the requirement $\xi_{1} \neq \xi_{2}$ is meant in the strong sense that we have the inequality

$$
\xi_{1}(\omega) \neq \xi_{2}(\omega) \text { for all } \omega \in \Omega,
$$

if at least one of the two values is finite. I.e. there shall not be a simultaneous occurrence of the two stresses, but $\xi_{1}(\omega)=\xi_{2}(\omega)=\infty$ should be possible. A central challenge of the multi-type stress worst-case portfolio problem is the unknown sequential occurrence of the different types of stresses. The investor not only has the difficulty that he/she does not know when the next stress will occur, but also which of the remaining stresses will occur. Still, we can draw on previous results and know the optimal portfolio process when at most one stress can occur. Thus, we can assume that we know

- the value function $v_{00}(t ; x)$ and the optimal sub strategy $\bar{\pi}_{(0,0)}=\pi^{*}$ if both stress scenarios have already happened (see Sect. 3),

- the value function $v_{10}(t ; x)$ and the optimal sub strategy $\bar{\pi}_{(1,0)}$ if only the stress scenario related to $z^{(2)}$ has already happened (see Sect. 4 with $(z, \xi)=\left(z^{(1)}, \xi_{1}\right)$ ),

- the value function $v_{01}(t ; x)$ and the optimal sub strategy $\bar{\pi}_{(0,1)}$ if only the stress scenario related to $z^{(1)}$ has already happened (see Sect. 4 with $(z, \xi)=\left(z^{(2)}, \xi_{2}\right)$ ).

Hence, it remains to determine the (worst-case) optimal sub strategy $\bar{\pi}_{(1,1)}$ and the value function $v_{11}(t ; x)$ if both stresses can still occur.

Reformulation of the problem. We now reformulate the problem such that we can at least partly use existing concepts. For this, we need to introduce the definition of the relative worst-case loss. This definition takes into account that for an admissible portfolio process at a certain point in time $t \in[0, T]$, the order in which the stresses occur can have different effects. In our worst-case approach, the order of occurrence that leads to a lower expected utility is of particular importance.

Definition 4 For a portfolio process $\pi$ we define the relative losses at time $t \in[0, T], R L_{12}^{\pi}(t)$ and $R L_{21}^{\pi}(t)$, depending on the order of stress occurrences, by

$$
\begin{aligned}
1-R L_{12}^{\pi}(t) & :=\left(1-\pi(t)^{T} z^{(1)}\right)\left(1-\bar{\pi}_{(0,1)}(t)^{T} z^{(2)}\right), \\
1-R L_{21}^{\pi}(t) & :=\left(1-\pi(t)^{T} z^{(2)}\right)\left(1-\bar{\pi}_{(1,0)}(t)^{T} z^{(1)}\right) .
\end{aligned}
$$

The relative worst-case loss $R L^{\pi}(t)$ of $\pi$ at time $t \in[0, T]$ is defined as

$$
R L^{\pi}(t):=\max \left\{R L_{12}^{\pi}(t), R L_{21}^{\pi}(t)\right\} .
$$


With $\Theta_{t}$ denoting the class of $[t, T] \cup\{\infty\}$-valued stopping times, we can define the value function $v_{11}(t ; x)$ by

$$
v_{11}(t ; x):=\sup _{\pi \in \mathbb{A}(t, x)} \inf _{\substack{\xi_{1}, \xi_{2} \in \Theta_{t} \\ \xi_{1} \neq \xi_{2}}} \mathbb{E}_{t, x,(1,1)}\left(U\left(X^{\pi}(T)\right)\right) \text { for } t \in[0, T],
$$

where we again use the strong notion of the inequality $\xi_{1} \neq \xi_{2}$ as in (5). Definition 4 allows to rewrite the value function $v_{11}(t ; x)$ in a suitable way.

Lemma 2 (Reformulation of the value function) The value function in the two-stress worst case problem can be rewritten as follows:

$$
\begin{aligned}
v_{11}(t ; x) & =\sup _{\pi \in \mathbb{A}(t, x)} \inf _{\substack{\xi_{1}, \xi_{2} \in \Theta_{t} \\
\xi_{1} \neq \xi_{2}}} \mathbb{E}_{t, x,(1,1)}\left(U\left(X^{\pi}(T)\right)\right) \\
& =\sup _{\pi \in \mathbb{A}(t, x)} \inf _{\xi \in \Theta_{t}} \mathbb{E}_{t, x}\left(v _ { 0 0 } \left(\xi ;\left(1-R L^{\left.\left.\left.\pi_{(1,1)}(\xi)\right) X^{\pi_{(1,1)}}(\xi-)\right)\right),}\right.\right.\right.
\end{aligned}
$$

where $v_{00}\left(\infty ;\left(1-R L^{\pi_{(1,1)}}(\infty)\right) X^{\pi_{(1,1)}}(\infty-)\right):=U\left(X^{\pi_{(1,1)}}(T)\right)$.

Proof The idea of this proof is to reduce the worst-case problem to a single stopping time $\xi$, instead of $\xi_{1}$ and $\xi_{2}$, by taking advantage of the fact that we know the optimal portfolio process after the first stress has occurred. To do this, we first have to introduce some notations:

- We define

$$
\begin{aligned}
& \tilde{\mathbb{A}}(t, x):=\left\{\tilde{\pi} \mid \tilde{\pi}(s)=\pi_{(1,1)}(s) \mathbb{1}_{\left\{s \leq \min \left\{\xi_{1}, \xi_{2}\right\}\right\}}+\bar{\pi}_{(1,0)}(s) \mathbb{1}_{\left\{\xi_{2}<s \leq \xi_{1}\right\}}\right. \\
& \left.+\bar{\pi}_{(0,1)}(s) \mathbb{1}_{\left\{\xi_{1}<s \leq \xi_{2}\right\}}+\pi^{*} \mathbb{1}_{\left\{s>\max \left\{\xi_{1}, \xi_{2}\right\}\right\}} \text { for } s \in[t, T] \text { and } \pi \in \mathbb{A}(t, x)\right\},
\end{aligned}
$$

as a subset of $\mathbb{A}(t, x)$.

- Further, we denote by $\tilde{\xi}=\min \left\{\xi_{1}, \xi_{2}\right\}$, for $\xi_{1}, \xi_{2} \in \Theta_{t}$, the time of occurrence of the first stress.

- Then, we define $\mathbb{E}_{\tilde{\xi}, x, 2}$ as the conditional expectation, given that $X^{\pi}(\tilde{\xi})=x$ and that the second stress can still occur, i.e. $\mathbb{E}_{\tilde{\xi}, x, 2}=\mathbb{E}_{\tilde{\xi}, x,(0,1)}$ if $\tilde{\xi}=\xi_{1}$ and $\mathbb{E}_{\tilde{\xi}, x, 2}=\mathbb{E}_{\tilde{\xi}, x,(1,0)}$ otherwise.

Due to the definition of $v_{00}\left(\infty ;\left(1-R L^{\pi_{(1,1)}}(\infty)\right) X^{\pi_{(1,1)}}(\infty-)\right)$, we only need to consider the case $\tilde{\xi}<\infty$. Using the introduced notation leads to

$$
\begin{aligned}
& \sup _{\pi \in \mathbb{A}(t, x)} \inf _{\substack{\xi_{1}, \xi_{2} \in \Theta_{t} \\
\xi_{1} \neq \xi_{2}}} \mathbb{E}_{t, x,(1,1)}\left(U\left(X^{\pi}(T)\right)\right) \\
&= \sup _{\pi \in \mathbb{A}(t, x)} \inf _{\substack{\xi_{1}, \xi_{2} \in \Theta_{t} \\
\xi_{1} \neq \xi_{2}}} \mathbb{E}_{t, x,(1,1)}\left(\mathbb{E}_{\tilde{\xi}, X^{\pi}(\tilde{\xi}), 2}\left(U\left(X^{\pi}(T)\right)\right)\right) \\
&= \sup _{\pi \in \tilde{\mathbb{A}}(t, x)} \inf _{\substack{\xi_{1}, \xi_{2} \in \Theta_{t} \\
\xi_{1} \neq \xi_{2}}} \mathbb{E}_{t, x,(1,1)}\left(\mathbb{E}_{\tilde{\xi}, X^{\pi}(\tilde{\xi}), 2}\left(U\left(X^{\pi}(T)\right)\right)\right) \\
&= \sup _{\pi \in \mathbb{A}(t, x)} \inf _{\substack{\xi_{1}, \xi_{2} \in \Theta_{t} \\
\xi_{1} \neq \xi_{2}}} \mathbb{E}_{t, x,(1,1)}\left(\mathbb{1}_{\tilde{\xi}=\xi_{1}} \mathbb{E}_{\tilde{\xi}, X^{\pi}(\tilde{\xi}),(0,1)}\left(U\left(X^{\pi}(T)\right)\right)\right. \\
&\left.\quad+\mathbb{1}_{\tilde{\xi}=\xi_{2}} \mathbb{E}_{\tilde{\xi}, X^{\pi}(\tilde{\xi}),(1,0)}\left(U\left(X^{\pi}(T)\right)\right)\right)
\end{aligned}
$$




$$
\begin{array}{r}
=\sup _{\pi \in \tilde{\mathbb{A}}(t, x)} \inf _{\substack{\xi_{1}, \xi_{2} \in \Theta_{t} \\
\xi_{1} \neq \xi_{2}}} \mathbb{E}_{t, x,(1,1)}\left(\mathbb{1}_{\tilde{\xi}=\xi_{1}} v_{01}\left(\tilde{\xi} ;\left(1-\pi_{(1,1)}(\tilde{\xi})^{T} z^{(1)}\right) X^{\pi_{(1,1)}}(\tilde{\xi}-)\right)\right. \\
\left.+\mathbb{1}_{\tilde{\xi}=\xi_{2}} v_{10}\left(\tilde{\xi} ;\left(1-\pi_{(1,1)}(\tilde{\xi})^{T} z^{(2)}\right) X^{\pi_{(1,1)}}(\tilde{\xi}-)\right)\right) .
\end{array}
$$

The first equality follows by the tower property. For the second equality note that in Sects. 3 and 4 we have already determined the optimal strategies for the different cases when at most one or even no more stress can occur. Thus, an optimal strategy in the case when at most two stresses can still occur, must have the form of the strategies in the set $\tilde{\mathbb{A}}(t, x)$, i.e. we can restrict to this set for determining the optimal strategies. The third equality is a direct result of the above introduced notation for the conditional expectation and the assumption $\xi_{1} \neq \xi_{2}$. Theorem 1 and the definition of $\widetilde{\mathbb{A}}(t, x)$, as well as the definition of the wealth process at time $\tilde{\xi}$, lead to the fourth equation.

Note that the reformulated value function now only depends on the time of occurrence of the first stress and because of $\xi_{1}, \xi_{2} \in \Theta_{t}$, this can be any stopping time in $\Theta_{t}$. Furthermore it follows from the definition of $\tilde{\xi}$ that

$$
\tilde{\xi}= \begin{cases}\xi_{1} & \text { if } \quad v_{01}\left(\xi_{1} ; X^{\pi}\left(\xi_{1}\right)\right) \leq v_{10}\left(\xi_{2} ; X^{\pi}\left(\xi_{2}\right)\right), \\ \xi_{2} & \text { else. }\end{cases}
$$

Thus we obtain

$$
\begin{aligned}
& \sup _{\pi \in \tilde{\mathbb{A}}(t, x)} \inf _{\substack{\xi_{1}, \xi_{2} \in \Theta_{t} \\
\xi_{1} \neq \xi_{2}}} \mathbb{E}_{t, x,(1,1)}\left(\mathbb{1}_{\tilde{\xi}=\xi_{1}} v_{01}\left(\tilde{\xi} ;\left(1-\pi_{(1,1)}(\tilde{\xi})^{T} z^{(1)}\right) X^{\pi_{(1,1)}}(\tilde{\xi}-)\right)\right. \\
& \left.+\mathbb{1}_{\tilde{\xi}=\xi_{2}} v_{10}\left(\tilde{\xi} ;\left(1-\pi_{(1,1)}(\tilde{\xi})^{T} z^{(2)}\right) X^{\pi_{(1,1)}}(\tilde{\xi}-)\right)\right) \\
& =\sup _{\pi \in \tilde{\mathbb{A}}(t, x)} \inf _{\xi \in \Theta_{t}} \mathbb{E}_{t, x}\left(\operatorname { m i n } \left\{v_{01}\left(\xi ;\left(1-\pi_{(1,1)}(\xi)^{T} z^{(1)}\right) X^{\pi_{(1,1)}}(\xi-)\right)\right.\right. \text {, } \\
& \left.\left.v_{10}\left(\xi ;\left(1-\pi_{(1,1)}(\xi)^{T} z^{(2)}\right) X^{\pi_{(1,1)}}(\xi-)\right)\right\}\right) \\
& =\sup _{\pi \in \tilde{\mathbb{A}}(t, x)} \inf _{\xi \in \Theta_{t}} \mathbb{E}_{t, x}\left(\operatorname { m i n } \left\{v_{00}\left(\xi ;\left(1-R L_{12}^{\pi_{(1,1)}}(\xi)\right) X^{\pi_{(1,1)}}(\xi-)\right)\right.\right. \text {, } \\
& \left.\left.v_{00}\left(\xi ;\left(1-R L_{21}^{\pi_{(1,1)}}(\xi)\right) X^{\pi_{(1,1)}}(\xi-)\right)\right\}\right) \\
& =\sup _{\pi \in \tilde{\mathbb{A}}(t, x)} \inf _{\xi \in \Theta_{t}} \mathbb{E}_{t, x}\left(\operatorname { m i n } \left\{\left(1-R L_{12}^{\pi_{(1,1)}}(\xi)\right)^{1-\gamma} v_{00}\left(\xi ; X^{\pi_{(1,1)}}(\xi-)\right)\right.\right. \text {, } \\
& \left.\left.\left(1-R L_{21}^{\pi_{(1,1)}}(\xi)\right)^{1-\gamma} v_{00}\left(\xi ; X^{\pi_{(1,1)}}(\xi-)\right)\right\}\right) \\
& =\sup _{\pi \in \tilde{\mathbb{A}}(t, x)} \inf _{\xi \in \Theta_{t}} \mathbb{E}_{t, x}\left(\left(1-R L^{\pi_{(1,1)}}(\xi)\right)^{1-\gamma} v_{00}\left(\xi ; X^{\pi_{(1,1)}}(\xi-)\right)\right) \\
& =\sup _{\pi \in \tilde{\mathbb{A}}(t, x)} \inf _{\xi \in \Theta_{t}} \mathbb{E}_{t, x}\left(v_{00}\left(\xi ;\left(1-R L^{\pi_{(1,1)}}(\xi)\right) X^{\pi_{(1,1)}}(\xi-)\right)\right) \\
& =\sup _{\pi \in \mathbb{A}(t, x)} \inf _{\xi \in \Theta_{t}} \mathbb{E}_{t, x}\left(v_{00}\left(\xi ;\left(1-R L^{\pi_{(1,1)}}(\xi)\right) X^{\pi_{(1,1)}}(\xi-)\right)\right) .
\end{aligned}
$$

Here, the first equality follows with the above considerations on $\tilde{\xi}$ and the second one with Theorem 1 and the definition of the relative losses $R L_{12}^{\pi_{(1,1)}}$ and $R L_{21}^{\pi_{(1,1)}}$. Equation three holds by Proposition 2 and for the fourth equality note that $v_{00}\left(t ; X^{\pi_{(1,1)}}(\xi-)\right)$ is negative for $\gamma>1$ and positive for $\gamma<1$ and for an admissible portfolio process it holds $R L_{12}^{\pi_{(1,1)}}<1$ 
and $R L_{21}^{\pi_{(1,1)}}<1$. The second but last equality follows again with Proposition 2 and the last one holds by the definition of $\tilde{\mathbb{A}}(t, x)$.

Remark 3 (Admissible portfolio processes) From now on, we denote, without loss of generality, by $\mathbb{A}(t, x)$, for $x>0, t \in[0, T]$, the reduced set of admissible portfolio processes $\tilde{\mathbb{A}}(t, x)$, defined in the proof of Lemma 2 .

Remark 4 (Wealth process before the first stress) At this point we would like to note that the stopping time $\xi \in \Theta$, in the reformulated representation of the value function, is the time of occurrence of the first stress. Thus, the wealth process cannot jump at a time $t<\xi$. This is of particular importance when using Itô's rule in the proofs of Proposition 5 and Proposition 8.

Minimum constant processes and optimality. The main consequence of Lemma 2 is that we only have to consider the infimum over a single stopping time $\xi \in \Theta$ in the value function $v_{11}(t ; x)$. This allows to suitably adapt the indifference optimality principle and the indifference frontier for the two-stress worst-case problem via the introduction of the concept of the minimum process.

Definition 5 Let $\pi \in \mathbb{A}(x)$ be an admissible portfolio process.

1. We define the minimum process $M^{\pi}(t)$ by

$$
M^{\pi}(t):=v_{00}\left(t ;\left(1-R L^{\pi_{(1,1)}}(t)\right) X^{\pi_{(1,1)}}(t-)\right) \text { for } t \in[0, T],
$$

with $M^{\pi}(\infty):=U\left(X^{\pi(1,1)}(T)\right)$.

2 . We call $\pi$ minimum constant if we have

$$
\mathbb{E}\left(M^{\pi}\left(\tau_{1}\right)\right)=\mathbb{E}\left(M^{\pi}\left(\tau_{2}\right)\right) \text { for all } \tau_{1}, \tau_{2} \in \Theta .
$$

The unknown order of appearance of the two stresses requires that the investor always has to consider both possible orderings simultaneously when judging a portfolio strategy in terms of the worst-case approach. For this reason, we have introduced the definition of the minimum process. The wording minimum process gets its meaning by noting that we have

$$
\begin{aligned}
& M^{\pi}(t)=\min \left\{v_{01}\left(t ;\left(1-\pi_{(1,1)}(t)^{T} z^{(1)}\right) X^{\pi_{(1,1)}}(t-)\right),\right. \\
&\left.v_{10}\left(t ;\left(1-\pi_{(1,1)}(t)^{T} z^{(2)}\right) X^{\pi_{(1,1)}}(t-)\right)\right\} \text { for } t \in[0, T],
\end{aligned}
$$

with the same arguments as in the proof of Lemma 2. To be able to argue similarly as for the indifference optimality, we also need minimum constant portfolio processes.

Remark 5 (Connection to the existing literature) In the previous definition we have introduced minimum constant portfolio processes. In addition, we will state the minimum constantoptimality principle in Proposition 6 and introduce the minimum indifference frontier in Proposition 7 and Remark 6.

To solve the worst-case scenario portfolio problem with a single stress, the notions of indifference strategy and indifference frontier have been introduced in [11] and [20]. These indifference concepts are based on an abstract controller-vs-stopper game between the investor (controller) and the market (stopper).

However there exists a fundamental difference, that clearly distinguishes our new concepts of minimum constant portfolio processes and of the minimum constant-optimality principle from the existing literature. For this, consider the one stress scenario, or even the portfolio optimization problem with $n>1$ stress scenarios, consisting of $n$ equal stresses $z_{1}=\ldots=$ 
$z_{n}$, as solved in [12]. In these problems, the investor chooses the portfolio process and the market decides when the next stress occurs. In our work, the investor still only has the option of choosing the portfolio process. The market, as his/her counterpart, now has the possibility to decide when the next stress will occur as well as which of the remaining stresses occurs. Thus, the market has two controls, namely the time and the order of occurrence of the stresses.

The existing concepts of indifference strategies and the indifference optimality principle are based on the idea that an optimal investor should be indifferent to the time of occurrence of the next stress, and at the same time presupposes the knowledge of which stress will be the next. It is exactly this knowledge that we can no longer assume in this work with multiple different stresses, which is why the introduction of new concepts is necessary. The new principle of minimum indifference is now intended to take into account an indifference with regard to the time of occurrence of the worst possible order of stresses. The formal implementation of these new concepts, which finally results in the proof of existence of an optimal portfolio process, is described in the remainder of this section.

We are already in a position, where we can prove the existence of a minimum constant portfolio process.

Proposition 5 (Existence of a minimum constant portfolio process) There exists a minimum constant portfolio process $\bar{\pi} \in \mathbb{A}(x)$, such that

$$
\bar{\pi}_{(1,1)}(t) \in \arg \max \underset{R L^{\pi}(t)=N(t)}{\pi \in \mathbb{R}^{2}} \underset{\gamma}{\operatorname{ar}(\pi)} \text { for } t \in[0, T],
$$

where $N$ is defined for $t \in[0, T]$ by the differential equation

$$
N^{\prime}(t)=(1-N(t))\left(\max _{\substack{\pi \in \mathbb{R}^{2} \\ R L^{\pi}(t)=N(t)}} \phi_{\gamma}(\pi)-\phi_{\gamma}\left(\pi^{*}\right)\right),
$$

with $N(T)=0$ and $\phi_{\gamma}$ as defined in Proposition 1. Further, for all $t \in[0, T]$ we have $R L^{\bar{\pi}_{(1,1)}}(t)<1$.

Proof We give the proof in two steps.

(i) First, we show the existence of a deterministic admissible portfolio process $\bar{\pi}$, as claimed in the assertion. We consider the function

$$
G(t, N):=\left(\max _{\substack{\pi \in \mathbb{R}^{2} \\ R L^{\pi}(t)=N}} \phi_{\gamma}(\pi)-\phi_{\gamma}\left(\pi^{*}\right)\right) .
$$

As $\phi_{\gamma}(\pi)$ is a strictly concave quadratic function in $\pi$ and $R L^{\pi}(t)$ is the maximum of two linear functions in $\pi$ with a range of $\mathbb{R}$, the maximization problem inside $G(t, N)$ can be solved and the maximum is attained. As further $R L^{\pi}(t)$ is continuous, both in $t$ and in $\pi, G(t, N)$ is also continuous. Thus, the ordinary differential equation (6) is well-defined. Now we can apply Lemma 4.2. in [20] and get, for the solution $N$ of (6), the inequality $0 \leq N(t)<1$ for all $t \in[0, T]$. Then it follows directly that a deterministic admissible portfolio process $\bar{\pi} \in \mathbb{A}(x)$, as defined above, exists and in particular $R L^{\bar{\pi}_{(1,1)}}=N$ applies for this portfolio process.

It is admissible as $N$ is continuous and bounded, $\phi_{\gamma}$ is a quadratic function with a 
unique maximum and therefore there exists a $\bar{\pi}_{(1,1)}$, which is continuous and bounded. Further, we get for every $t \in[0, T]$

$$
\begin{aligned}
N(t)<1 \Leftrightarrow & R L^{\bar{\pi}_{(1,1)}}(t)<1 \Leftrightarrow \max \left\{R L_{12}^{\bar{\pi}_{(1,1)}}(t), R L_{21}^{\bar{\pi}_{(1,1)}}(t)\right\}<1 \\
\Leftrightarrow & 1-\left(1-\bar{\pi}_{(1,1)}(t)^{T} z^{(1)}\right)\left(1-\bar{\pi}_{(0,1)}(t)^{T} z^{(2)}\right)<1 \\
& \wedge 1-\left(1-\bar{\pi}_{(1,1)}(t)^{T} z^{(2)}\right)\left(1-\bar{\pi}_{(1,0)}(t)^{T} z^{(1)}\right)<1 \\
\Leftrightarrow & \max \left\{\bar{\pi}_{(1,1)}(t)^{T} z^{(1)}, \bar{\pi}_{(1,1)}(t)^{T} z^{(2)}\right\}<1,
\end{aligned}
$$

where the last equivalence is valid since $\bar{\pi}_{(1,0)}$ and $\bar{\pi}_{(0,1)}$ are part of admissible portfolio processes in the one-stress setting and thus $\bar{\pi}_{(1,0)}(t)^{T} z^{(1)}<1$ and $\bar{\pi}_{(0,1)}(t)^{T} z^{(2)}<1$.

(ii) Now we prove the minimum constant property of an admissible portfolio process $\bar{\pi}$, defined as above. For $t \in[0, T]$, the minimum process $M^{\bar{\pi}}$ is defined as

$$
M^{\bar{\pi}}(t)=v_{00}\left(t ;\left(1-R L^{\bar{\pi}_{(1,1)}}(t)\right) X^{\bar{\pi}_{(1,1)}}(t-)\right) .
$$

By definition of $\bar{\pi}$ we have

$$
\left(R L^{\bar{\pi}_{(1,1)}}\right)^{\prime}(t)=\left(1-R L^{\left.\bar{\pi}_{(1,1)}(t)\right)}\left(\phi_{\gamma}\left(\bar{\pi}_{(1,1)}(t)\right)-\phi_{\gamma}\left(\pi^{*}\right)\right),\right.
$$

with $R L^{\bar{\pi}_{(1,1)}}(T)=0$. Itô's rule for $v_{00}\left(t ;\left(1-R L^{\left.\left.\bar{\pi}_{(1,1)}(t)\right) X^{\bar{\pi}_{(1,1)}}(t-)\right) \text { leads to }}\right.\right.$

$$
\begin{aligned}
& d v_{00}\left(t ;\left(1-R L^{\left.\left.\bar{\pi}_{(1,1)}(t)\right) X^{\bar{\pi}_{(1,1)}}(t-)\right)}\right.\right. \\
= & \left.\left.\left(v_{00}\right)_{t}(t ; x(t))\right) d t+\left(v_{00}\right)_{x}(t ; x(t))\right) x(t)\left(r+\bar{\pi}_{(1,1)}(t)^{T}(b-r \underline{1})\right) d t \\
& \left.-\left(v_{00}\right)_{x}(t ; x(t))\right) x(t) \frac{\left(R L^{\bar{\pi}_{(1,1)}}\right)^{\prime}(t)}{1-R L^{\bar{\pi}_{(1,1)}}(t)} d t \\
& \left.+\frac{1}{2}\left(v_{00}\right)_{x x}(t ; x(t))\right) x(t)^{2} \bar{\pi}_{(1,1)}(t)^{T} \sigma \sigma^{T} \bar{\pi}_{(1,1)}(t) d t \\
& \left.+\left(v_{00}\right)_{x}(t ; x(t))\right) x(t) \bar{\pi}_{(1,1)}(t)^{T} \sigma d W(t),
\end{aligned}
$$

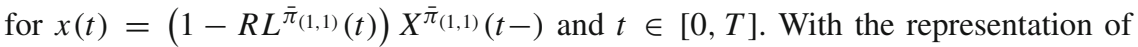
$v_{00}(t ; x)$ from Proposition 2 and Eq. (7) we get

$$
d v_{00}(t ; x(t))=v_{00}(t ; x(t))(1-\gamma) \bar{\pi}_{(1,1)}(t)^{T} \sigma d W(t) .
$$

The solution of this SDE is given by

$$
\begin{aligned}
& v_{00}\left(t ;\left(1-R L^{\bar{\pi}_{(1,1)}}(t)\right) X^{\bar{\pi}_{(1,1)}(t-)}\right)
\end{aligned}
$$

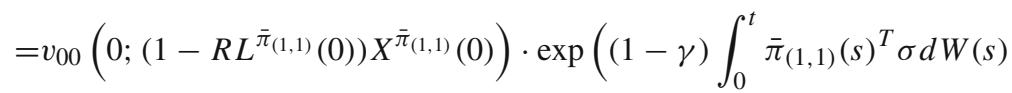

$$
\begin{aligned}
& \left.-\frac{1}{2}(1-\gamma)^{2} \int_{0}^{t} \bar{\pi}_{(1,1)}(s)^{T} \sigma \sigma^{T} \bar{\pi}_{(1,1)}(s) d s\right),
\end{aligned}
$$

for $t \in[0, T]$. Together with the integrability assumptions of an admissible portfolio process and Novikov's condition we get that

$$
v_{00}\left(t ;\left(1-R L^{\bar{\pi}_{(1,1)}}(t)\right) X^{\bar{\pi}_{(1,1)}}(t-)\right)
$$


is a martingale on $[0, T]$. The boundary condition $R L^{\pi_{(1,1)}}(T)=0$ extends the martingale property for $M^{\bar{\pi}}$ to $[0, T] \cup\{\infty\}$. By Doob's optional sampling theorem the stopped process is also a martingale and so we get the minimum constant property on $[0, T] \cup\{\infty\}$.

Before we prove the optimality of such a minimum constant portfolio process, we introduce two helpful concepts. First, as analogue to the indifference-optimality we formulate the minimum constant-optimality principle.

Proposition 6 (Minimum constant-optimality principle) If $\bar{\pi} \in \mathbb{A}(x)$ is a minimum constant portfolio process and for all $\pi \in \mathbb{A}(x)$ we have

$$
M^{\bar{\pi}}(\tilde{\xi}) \geq M^{\pi}(\tilde{\xi}) \text { for at least one } \tilde{\xi} \in \Theta,
$$

then $\bar{\pi}$ solves the worst-case portfolio problem under stresses (5).

Proof The definition of a minimum constant portfolio process implies:

$$
\inf _{\xi \in \Theta} \mathbb{E}\left(M^{\bar{\pi}}(\xi)\right)=\mathbb{E}\left(M^{\bar{\pi}}(\tilde{\xi})\right) \geq \mathbb{E}\left(M^{\pi}(\tilde{\xi})\right) \geq \inf _{\xi \in \Theta} \mathbb{E}\left(M^{\pi}(\xi)\right),
$$

and together with Lemma 2 the claim is proven.

Next, we construct a minimum indifference frontier for the two-stress worst-case problem so that all portfolio processes which exceed this frontier cannot be optimal for the worst-case problem.

Proposition 7 (Minimum indifference frontier) Let $\pi \in \mathbb{A}(x)$, and let $\bar{\pi} \in \mathbb{A}(x)$ be a minimum constant portfolio process. Set

$$
\begin{aligned}
& \tau_{1}:=\inf \left\{t: R L_{12}^{\pi_{(1,1)}}(t)>R L^{\bar{\pi}_{(1,1)}}(t)\right\}, \\
& \tau_{2}:=\inf \left\{t: R L_{21}^{\pi_{(1,1)}}(t)>R L^{\bar{\pi}_{(1,1)}}(t)\right\},
\end{aligned}
$$

and define

$$
\begin{aligned}
& { }_{1} \tilde{\pi}_{(1,1)}(t):=\pi_{(1,1)}(t) \text { if } t<\tau_{1} \text { and }{ }_{1} \tilde{\pi}_{(1,1)}(t):=\bar{\pi}_{(1,1)}(t) \text { if } t \geq \tau_{1}, \\
& { }_{2} \tilde{\pi}_{(1,1)}(t):=\pi_{(1,1)}(t) \text { if } t<\tau_{2} \text { and }{ }_{2} \tilde{\pi}_{(1,1)}(t):=\bar{\pi}_{(1,1)}(t) \text { if } t \geq \tau_{2} .
\end{aligned}
$$

Then, ${ }_{1} \tilde{\pi},{ }_{2} \tilde{\pi} \in \mathbb{A}(x)$ and the worst-case performances in (5) attained by ${ }_{1} \tilde{\pi}$ and ${ }_{2} \tilde{\pi}$ are at least as big as that achieved by $\pi$.

Proof We give the proof for ${ }_{1} \tilde{\pi}$ and $\tau_{1}$. The proof for ${ }_{2} \tilde{\pi}$ follows analogously. First note that ${ }_{1} \tilde{\pi}_{(1,1)}$ is right-continuous by construction. Further, $\tau_{1}$ is predictable and therefore also ${ }_{1} \tilde{\pi}_{(1,1)}$ is predictable. Thus we have ${ }_{1} \tilde{\pi} \in \mathbb{A}(x)$.

Now it is enough to look at $\tau_{1}<\infty$ as otherwise we directly get $M^{\pi}(t)=M^{1 \tilde{\pi}}(t)$ for all $t \in[0, T] \cup\{\infty\}$. By definition we have ${ }_{1} \tilde{\pi}_{(1,1)}(t)=\pi_{(1,1)}(t)$ if $0 \leq t<\tau_{1}$, and as $\bar{\pi}$ is minimum constant, we get that $\tilde{\pi}$ is minimum constant for $\left[\tau_{1}, T\right] \cup\{\infty\}$-valued stopping times. Furthermore, the construction of ${ }_{1} \tilde{\pi}$ and $\tau_{1}$ leads to

$$
M^{\pi}(t)=M^{1 \tilde{\pi}}(t) \text { for } t \in\left[0, \tau_{1}\right)
$$

and for $t=\tau_{1}$ : 


$$
\begin{aligned}
& \mathbb{E}\left(M^{\pi}\left(\tau_{1}\right)\right)=\mathbb{E}\left(v_{00}\left(\tau_{1} ;\left(1-R L^{\pi_{(1,1)}}\left(\tau_{1}\right)\right) X^{\pi_{(1,1)}}\left(\tau_{1}-\right)\right)\right) \\
& =\mathbb{E}\left(\operatorname { m i n } \left\{v_{00}\left(\tau_{1} ;\left(1-R L_{12}^{\pi_{(1,1)}}\left(\tau_{1}\right)\right) X^{\pi_{(1,1)}}\left(\tau_{1}-\right)\right)\right.\right. \text {, } \\
& \left.\left.v_{00}\left(\tau_{1} ;\left(1-R L_{21}^{\pi_{(1,1)}}\left(\tau_{1}\right)\right) X^{\pi_{(1,1)}}\left(\tau_{1}-\right)\right)\right\}\right) \\
& \leq \mathbb{E}\left(\operatorname { m i n } \left\{v_{00}\left(\tau_{1} ;\left(1-R L^{\bar{\pi}_{(1,1)}}\left(\tau_{1}\right)\right) X^{\pi_{(1,1)}}\left(\tau_{1}-\right)\right),\right.\right. \\
& \left.\left.v_{00}\left(\tau_{1} ;\left(1-R L_{21}^{\pi_{(1,1)}}\left(\tau_{1}\right)\right) X^{\pi_{(1,1)}}\left(\tau_{1}-\right)\right)\right\}\right) \\
& \leq \mathbb{E}\left(v_{00}\left(\tau_{1} ;\left(1-R L^{\bar{\pi}_{(1,1)}}\left(\tau_{1}\right)\right) X^{\pi_{(1,1)}}\left(\tau_{1}-\right)\right)\right)=\mathbb{E}\left(M^{1 \tilde{\pi}}\left(\tau_{1}\right)\right) .
\end{aligned}
$$

The second equality follows from the proof of Lemma 2 . The first inequality holds, since $v_{00}(t ; x)$ is monotonically increasing in $x$ and by the right-continuity properties of admissible portfolio processes. Let $\Theta^{\tau_{1}}$ denote the set of $\left[0, \tau_{1}\right]$-valued stopping times. Then, we obtain

$$
\inf _{\xi \in \Theta} \mathbb{E}\left(M^{1 \tilde{\pi}}(\xi)\right)=\inf _{\xi \in \Theta^{\tau_{1}}} \mathbb{E}\left(M^{1^{\tilde{\pi}}}(\xi)\right) \geq \inf _{\xi \in \Theta^{\tau_{1}}} \mathbb{E}\left(M^{\pi}(\xi) \geq \inf _{\xi \in \Theta} \mathbb{E}\left(M^{\pi}(\xi)\right),\right.
$$

and the claim follows with Lemma 2.

Remark 6 (Minimum indifference frontier) We briefly discuss the meaning of the two previous Propositions and the concept of the indifference frontier. For a minimum constant portfolio process $\bar{\pi}$ we define the set $I D(\bar{\pi})$ of all admissible portfolio processes that respect the minimum indifference frontier of $\bar{\pi}$ as follows:

$$
I D(\bar{\pi}):=\left\{\pi \in \mathbb{A}(x) \mid R L^{\pi_{(1,1)}}(t) \leq R L^{\bar{\pi}_{(1,1)}}(t) \text { for all } t \in[0, T]\right\} .
$$

From Proposition 7 we conclude that we can limit the search for the optimal strategy to $I D(\bar{\pi})$ and from Proposition 6 we know that it is enough to show that the minimum constant portfolio process $\bar{\pi}$ is the best strategy in $I D(\bar{\pi})$ for the no-stress scenario.

In Theorem 2 we state the existence of an optimal minimum constant portfolio process. Its proof is based on the following two lemmas.

Lemma 3 (Left-hand derivative of $R L^{\pi^{*}}$ ) Let $\pi^{*}$ be the optimal post stress portfolio process. Then the left-hand derivative of the relative worst-case loss $R L^{\pi^{*}}$ exists and we obtain:

$$
\lim _{\substack{h \rightarrow 0 \\ h<0}} \frac{R L^{\pi^{*}}(t+h)-R L^{\pi^{*}}(t)}{h}<0,
$$

for all $t \in(0, T]$, if $\max \left\{\left(\pi^{*}\right)^{T} z^{(1)},\left(\pi^{*}\right)^{T} z^{(2)}\right\}<1$.

Proof We know that $R L^{\pi^{*}}$ is the maximum of the two functions $R L_{12}^{\pi^{*}}$ and $R L_{21}^{\pi^{*}}$ with derivatives given by (see 1)

$$
\begin{aligned}
& \left(R L_{12}^{\pi^{*}}\right)^{\prime}(t)=\left(1-\left(\pi^{*}\right)^{T} z^{(1)}\right)\left(\bar{\pi}_{(0,1)}^{T} z^{(2)}\right)^{\prime}(t)<0, \\
& \left(R L_{21}^{\pi^{*}}\right)^{\prime}(t)=\left(1-\left(\pi^{*}\right)^{T} z^{(2)}\right)\left(\bar{\pi}_{(1,0)}^{T} z^{(1)}\right)^{\prime}(t)<0, \\
& \left(R L_{12}^{\pi^{*}}\right)^{\prime \prime}(t)=\left(1-\left(\pi^{*}\right)^{T} z^{(1)}\right)\left(\bar{\pi}_{(0,1)}^{T} z^{(2)}\right)^{\prime \prime}(t)<0, \\
& \left(R L_{21}^{\pi^{*}}\right)^{\prime \prime}(t)=\left(1-\left(\pi^{*}\right)^{T} z^{(2)}\right)\left(\bar{\pi}_{(1,0)}^{T} z^{(1)}\right)^{\prime \prime}(t)<0,
\end{aligned}
$$


for $t \in[0, T]$. If $R L_{12}^{\pi^{*}}(t)=R L_{21}^{\pi^{*}}(t)$ for all $t \in[0, T]$, the claim follows directly by the first derivatives. Otherwise we should note that $R L^{\pi^{*}}$ is the maximum of two strictly concave functions. By Lemma 2.11. in [2], the two functions have a maximum of two intersections. Thus $R L^{\pi^{*}}$ is differentiable from the left and together with the above first derivatives of $R L_{12}^{\pi^{*}}$ and $R L_{21}^{\pi^{*}}$, we obtain for the left-hand limit of the difference quotient:

$$
\lim _{\substack{h \rightarrow 0 \\ h<0}} \frac{R L^{\pi^{*}}(t+h)-R L^{\pi^{*}}(t)}{h}<0,
$$

for all $t \in(0, T]$.

Lemma 4 (Bounded relative worst-case loss) Let $\bar{\pi} \in \mathbb{A}(x)$ be a minimum constant portfolio process defined as in Proposition 5 and $\pi^{*}$ be the optimal post stress portfolio process. Then, for all $t \in[0, T]$ we get

$$
R L^{\bar{\pi}_{(1,1)}}(t) \leq R L^{\pi^{*}}(t) \text {, if } \max \left\{\left(\pi^{*}\right)^{T} z^{(1)},\left(\pi^{*}\right)^{T} z^{(2)}\right\}<1 .
$$

Proof First we revert time and define $F^{\bar{\pi}_{(1,1)}}(t)=R L^{\bar{\pi}_{(1,1)}}(T-t)$. Then we obtain the forward ODE of $R L^{\bar{\pi}_{(1,1)}}$ for $t \in[0, T]$ :

$$
\left(F^{\bar{\pi}_{(1,1)}}\right)^{\prime}(t)=-\left(1-F^{\left.\bar{\pi}_{(1,1)}(t)\right)}\left(\max _{\substack{\pi \in \mathbb{R}^{2} \\ \bar{\pi}_{(1,1)}(t)}} \phi_{\gamma}(\pi)-\phi_{\gamma}\left(\pi^{*}\right)\right),\right.
$$

with $F^{\bar{\pi}_{(1,1)}}(0)=0$. Further we define $F^{\pi^{*}}(t)=R L^{\pi^{*}}(T-t)$ for $t \in[0, T]$ and with Lemma 3 we obtain

$$
\lim _{\substack{h \rightarrow 0 \\ h>0}} \frac{F^{\pi^{*}}(t+h)-F^{\pi^{*}}(t)}{h}>0,
$$

for all $t \in[0, T)$. Now we have to show that $F^{\pi^{*}}(t) \geq F^{\bar{\pi}_{(1,1)}}(t)$ for $t \in[0, T]$. Assumption (2) leads to

$$
F^{\bar{\pi}_{(1,1)}}(0)=0<\max \left\{\left(\pi^{*}\right)^{T} z^{(1)},\left(\pi^{*}\right)^{T} z^{(2)}\right\}=F^{\pi^{*}}(0) .
$$

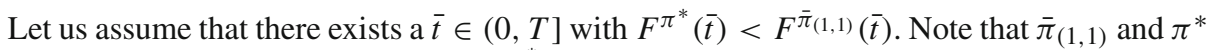
are continuous and so are $F^{\bar{\pi}_{(1,1)}}$ and $F^{\pi^{*}}$. Therefore, there is at least one point of intersection $t_{0} \in(0, \bar{t})$ and an $\epsilon>0$, such that $F^{\pi^{*}}\left(t_{0}\right)=F^{\bar{\pi}_{(1,1)}}\left(t_{0}\right)$ and

$$
F^{\pi^{*}}\left(t_{0}+h\right)-F^{\bar{\pi}_{(1,1)}}\left(t_{0}+h\right)<0 \text { for all } h \in(0, \epsilon]
$$

This leads to

$$
\lim _{\substack{h \rightarrow 0 \\ h>0}} \frac{\left(F^{\pi^{*}}\left(t_{0}+h\right)-F^{\bar{\pi}_{(1,1)}}\left(t_{0}+h\right)\right)-\left(F^{\pi^{*}}\left(t_{0}\right)-F^{\bar{\pi}_{(1,1)}}\left(t_{0}\right)\right)}{h} \leq 0 .
$$

Then, the differentiability of $F^{\bar{\pi}_{(1,1)}}$ implies

$$
\begin{aligned}
\lim _{\substack{h \rightarrow 0 \\
h>0}} \frac{F^{\pi^{*}}\left(t_{0}+h\right)-F^{\pi^{*}}\left(t_{0}\right)}{h} & \leq \lim _{\substack{h \rightarrow 0 \\
h>0}} \frac{F^{\bar{\pi}_{(1,1)}}\left(t_{0}+h\right)-F^{\bar{\pi}_{(1,1)}}\left(t_{0}\right)}{h} \\
& =\left(F^{\bar{\pi}_{(1,1)}}\right)^{\prime}\left(t_{0}\right) .
\end{aligned}
$$


However, this is a contradiction, as on one hand inequality (8) is valid and on the other hand, $\pi^{*}$ is the unconstrained maximizer of $\phi_{\gamma}$ which yields

$$
\begin{aligned}
& \left(F^{\bar{\pi}_{(1,1)}}\right)^{\prime}\left(t_{0}\right)=-\left(1-F^{\left.\bar{\pi}_{(1,1)}\left(t_{0}\right)\right)}\left(\max _{\substack{\pi \in \mathbb{R}^{2} \\
R L^{\pi}\left(T-t_{0}\right)=F^{\pi^{*}}\left(t_{0}\right)}} \phi_{\gamma}(\pi)-\phi_{\gamma}\left(\pi^{*}\right)\right)\right. \\
& =-\left(1-F^{\bar{\pi}_{(1,1)}}\left(t_{0}\right)\right)\left(\phi_{\gamma}\left(\pi^{*}\right)-\phi_{\gamma}\left(\pi^{*}\right)\right)=0 .
\end{aligned}
$$

So $F^{\bar{\pi}_{(1,1)}}(t) \leq F^{\pi^{*}}(t)$ and therefore $R L^{\bar{\pi}_{(1,1)}}(t) \leq R L^{\pi^{*}}(t)$ for all $t \in[0, T]$.

Theorem 2 (Solution of the multi-stress worst-case problem) Let $\bar{\pi} \in \mathbb{A}(x)$ be a minimum constant portfolio process defined as in Proposition 5. Then, $\bar{\pi}$ is a worst-case optimal portfolio process of problem (5). For the corresponding value function $v_{11}(t ; x)$, we get at time $t=0$ :

$$
v_{11}(0 ; x)=v_{00}\left(0 ;\left(1-R L^{\bar{\pi}_{(1,1)}}(0)\right) x\right) .
$$

Proof Let $\bar{\pi} \in \mathbb{A}(x)$ be a minimum constant portfolio process defined as in Proposition 5 . In step (i) of this proof we show that $R L^{\bar{\pi}_{(1,1)}}(t) \leq R L^{\pi^{*}}(t)$ for all $t \in[0, T]$. Then in step (ii) we prove that

$$
\bar{\pi} \in \arg \max _{\pi \in I D(\bar{\pi})} \mathbb{E}\left(M^{\pi}(\infty)\right)=\arg \max _{\pi \in I D(\bar{\pi})} \mathbb{E}\left(U\left(X^{\pi_{(1,1)}}(T)\right)\right),
$$

where $I D(\bar{\pi})$ is defined by the minimum indifference frontier as

$$
I D(\bar{\pi}):=\left\{\pi \in \mathbb{A}(x) \mid R L^{\pi_{(1,1)}}(t) \leq R L^{\bar{\pi}_{(1,1)}}(t) \text { for all } t \in[0, T]\right\} .
$$

Hence, $\bar{\pi}$ solves the portfolio optimization problem in the stress-free world in the set of all admissible portfolio processes respecting its indifference frontier. Together with Propositions 6 and 7 this directly yields optimality of $\bar{\pi}$.

(i) We first want to show that $R L^{\bar{\pi}_{(1,1)}}(t) \leq R L^{\pi^{*}}(t)$ for all $t \in[0, T]$. For this, we have to distinguish two cases.

(a) If $\max \left\{\left(\pi^{*}\right)^{T} z^{(1)},\left(\pi^{*}\right)^{T} z^{(2)}\right\} \geq 1$, we get $R L^{\pi^{*}}(t) \geq 1$ for all $t \in[0, T]$. But by Proposition 5 we know $R L^{\bar{\pi}_{(1,1)}(t)}<1$ for all $t \in[0, T]$ and therefore $R L^{\bar{\pi}_{(1,1)}}(t)<R L^{\pi^{*}}(t)$ for all $t \in[0, T]$.

(b) In the case of $\max \left\{\left(\pi^{*}\right)^{T} z^{(1)},\left(\pi^{*}\right)^{T} z^{(2)}\right\}<1$, Lemma 4 gives us the inequality.

(ii) Before we prove the optimality of $\bar{\pi}$, we show that $\bar{\pi}$ solves the constrained optimization problem

$$
\max _{\pi \in I D(\bar{\pi})} \phi_{\gamma}\left(\pi_{(1,1)}(t)\right) \text { for all } t \in[0, T] .
$$

We maximize the quadratic function $\phi_{\gamma}$ under linear constraints. From part (i) we know that the unique optimizer $\pi^{*}$ of $\phi_{\gamma}$ in the unconstrained case does not fulfill the constraints strictly, i.e. $R L^{\pi^{*}}(t) \nless R L^{\bar{\pi}_{(1,1)}}(t)<1$ for any $t \in[0, T]$. Thus, the optimizer $\pi^{c}$ of the constrained optimization problem (10) is a boundary point of $I D(\bar{\pi})$. That means $R L^{\pi_{(1,1)}^{c}}(t)=R L^{\bar{\pi}_{(1,1)}}(t)$ for all $t \in[0, T]$. The definition of $\bar{\pi}$ in Proposition 5 yields

$$
\bar{\pi}_{(1,1)}(t) \in \arg \max \underset{\substack{\pi \in \mathbb{R}^{2} \\ R L^{\pi}(t)=R L^{\bar{\pi}_{(1,1)}(t)}}}{ } \phi_{\gamma}(\pi) \text { for all } t \in[0, T],
$$


and thus $\bar{\pi}$ is a solution of (10).

Now we show (9) and hence prove the optimality of $\bar{\pi}$. For this, we apply the terminal utility decomposition of Proposition 1, i.e. for $\pi \in \mathbb{A}(x)$ we have in the no-stress scenario:

$$
U\left(X^{\pi_{(1,1)}}(T)\right)=U(x) \exp \left((1-\gamma) \int_{0}^{T} \phi_{\gamma}\left(\pi_{(1,1)}(t)\right) d t\right) Y_{T}^{\gamma}\left(\pi_{(1,1)}\right),
$$

where $Y^{\gamma}\left(\pi_{(1,1)}\right)=\left(Y_{t}^{\gamma}\left(\pi_{(1,1)}\right)\right)_{t \in[0, T]}$ is a positive $\mathcal{F}_{t}$-martingale with $Y_{0}^{\gamma}\left(\pi_{(1,1)}\right)=$ 1. Since $\bar{\pi}$ solves (10), we get for $\pi \in I D(\bar{\pi})$

$$
\begin{aligned}
& \mathbb{E}\left(M^{\pi}(\infty)\right)=\mathbb{E}\left(U\left(X^{\pi_{(1,1)}}(T)\right)\right) \\
& =\mathbb{E}\left(U(x) \exp \left((1-\gamma) \int_{0}^{T} \phi_{\gamma}\left(\pi_{(1,1)}(t)\right) d t\right) Y_{T}^{\gamma}\left(\pi_{(1,1)}\right)\right) \\
& \leq \mathbb{E}\left(U(x) \exp \left((1-\gamma) \int_{0}^{T} \phi_{\gamma}\left(\bar{\pi}_{(1,1)}(t)\right) d t\right) Y_{T}^{\gamma}\left(\pi_{(1,1)}\right)\right) \\
& =\mathbb{E}\left(U(x) \exp \left((1-\gamma) \int_{0}^{T} \phi_{\gamma}\left(\bar{\pi}_{(1,1)}(t)\right) d t\right) Y_{T}^{\gamma}\left(\bar{\pi}_{(1,1)}\right)\right)=\mathbb{E}\left(M^{\bar{\pi}}(\infty)\right) .
\end{aligned}
$$

Combining this with the arguments given at the beginning of this proof, we have shown that a minimum constant process exists which is a worst-case optimal portfolio process of problem (5).

Since $\bar{\pi}$ is a minimum constant portfolio process by construction, we get, together with Lemma 2:

$$
\begin{aligned}
v_{11}(0 ; x) & =\sup _{\pi \in \mathbb{A}(x)} \inf _{\xi \in \Theta} \mathbb{E}\left(M^{\pi}(\xi)\right)=\inf _{\xi \in \Theta} \mathbb{E}\left(M^{\bar{\pi}}(\xi)\right)=\mathbb{E}\left(M^{\bar{\pi}}(0)\right) \\
& =\mathbb{E}\left(v_{00}\left(0 ;\left(1-R L^{\bar{\pi}_{(1,1)}}(0)\right) x\right)\right)=v_{00}\left(0 ;\left(1-R L^{\bar{\pi}_{(1,1)}}(0)\right) x\right) .
\end{aligned}
$$

We would like to briefly point out that the previous concepts can be extended to the worst-case portfolio problem with more than two stresses and also to the case of a logarithmic utility function.

Remark 7 (Extension to more than two stresses) The recursive determination of the optimal strategies, depending on how much stresses can still occur, enables the extension to more than two stresses. The definitions from Sect. 5, as well as the minimum indifference frontier and the corresponding minimum indifference-optimality principle, can be adapted for the general $n$ stress case. Since we can define the relative worst-case loss completely analogous to Definition 4, the existence result in Proposition 5 as well as the proof of optimality in Theorem 2 can be used for the $n$ stress case.

Remark 8 (Logarithmic utility) The worst-case portfolio problem under stresses can be solved in the case of a logarithmic utility function, i.e. for $U(x)=\ln (x)$, with completely analogous proofs. Note that we obtain, with the help of Itô's formula, a decomposition of the terminal utility as in Proposition 1, which is even additive. Based on this, the classical Merton problem with random initial time can be solved. The one-stress worst-case problem in the multi asset setting is also treated in [9]. The proofs of Sect. 5 are quite analogous for the log-utility case, noting that the newly introduced concepts of the relative worst-case loss and minimum constant portfolio processes are defined independently of the concrete form of utility function. 


\section{Sub-scenarios and a verification theorem}

Proposition 2 and Theorem 1 allow us to construct the optimal strategy in the post stress situation and in the one-stress worst-case setting. In Theorem 2 we have shown that there exists a minimum constant portfolio process in the multi-stress case, which is optimal if both stresses can still occur. However, the proof of existence of this strategy, given in Proposition 5, is not constructive. Therefore, the goal is to derive a verification theorem, on the basis of which we then create an algorithmic framework, that enables us to construct the optimal strategy in a multi-stress worst-case scenario.

Sub-scenarios. We first define and determine indifference strategies for the sub-scenarios, when the order of occurrence of the stresses is known.

Definition 6 We consider a worst-case portfolio problem with two stress scenarios $\left(z^{(1)}, \xi_{1}\right)$ and $\left(z^{(2)}, \xi_{2}\right)$.

1. If there is a $\pi \in \mathbb{A}(x)$, such that

$$
v_{00}\left(t ;\left(1-R L_{12}^{\pi_{(1,1)}}(t)\right) X^{\pi_{(1,1)}}(t-)\right), t \in[0, T],
$$

is a martingale on $[0, T]$ with $R L_{12}^{\pi_{(1,1)}}(T)=0$, we call $\pi$ a sub-scenario indifference strategy for the sub-scenario $\xi_{1}<\xi_{2}$.

2. If there is a $\pi \in \mathbb{A}(x)$, such that

$$
v_{00}\left(t ;\left(1-R L_{21}^{\pi_{(1,1)}}(t)\right) X^{\pi_{(1,1)}}(t-)\right), t \in[0, T],
$$

is a martingale on $[0, T]$ with $R L_{21}^{\pi_{(1,1)}}(T)=0$, we call $\pi$ a sub-scenario indifference strategy for the sub-scenario $\xi_{2}<\xi_{1}$.

The required martingale property enables us to compute such sub-scenario indifference strategies.

Proposition 8 (Sub-scenario indifference strategy) For $\pi \in \mathbb{A}(x)$, define $f^{\pi_{(1,1)}}$ by

$$
f^{\pi_{(1,1)}}(t)=\pi_{(1,1)}(t)^{T}(b-r \underline{1})-\frac{1}{2} \gamma\left(\pi_{(1,1)}(t)^{T} \sigma \sigma^{T} \pi_{(1,1)}(t)+\left(\pi^{*}\right)^{T} \sigma \sigma^{T} \pi^{*}\right) .
$$

1. Let $\pi \in \mathbb{A}(x)$ and $\pi_{(1,1)}$ be a deterministic solution to the following one-dimensional coupled, non-linear ordinary differential equation

$$
\begin{aligned}
& \pi_{(1,1)^{\prime}(t)^{T} z^{(1)}} \\
= & \left(1-\pi_{(1,1)}(t)^{T} z^{(1)}\right)\left(f^{\left.\pi_{(1,1)}(t)-\frac{\bar{\pi}_{(0,1)}^{\prime}(t)^{T} z^{(2)}}{1-\bar{\pi}_{(0,1)}(t)^{T} z^{(2)}}\right),},\right.
\end{aligned}
$$

for $t \in[0, T]$ and with $\pi_{(1,1)}(T)^{T} z^{(1)}=0$. Then $\pi$ is a sub-scenario indifference strategy with respect to the sub-scenario $\xi_{1}<\xi_{2}$.

2. Let $\pi \in \mathbb{A}(x)$ and $\pi_{(1,1)}$ be a deterministic solution to the following one-dimensional coupled, non-linear ordinary differential equation

$$
\begin{aligned}
& \pi_{(1,1)^{\prime}(t)^{T} z^{(2)}} \\
= & \left(1-\pi_{(1,1)}(t)^{T} z^{(2)}\right)\left(f^{\left.\pi_{(1,1)}(t)-\frac{\bar{\pi}_{(1,0)}^{\prime}(t)^{T} z^{(1)}}{1-\bar{\pi}_{(1,0)}(t)^{T} z^{(1)}}\right),},\right.
\end{aligned}
$$


for $t \in[0, T]$ and with $\pi_{(1,1)}(T)^{T} z^{(2)}=0$. Then $\pi$ is a sub-scenario indifference strategy with respect to the sub-scenario $\xi_{2}<\xi_{1}$.

Proof We give the proof for $\pi \in \mathbb{A}(x)$, fulfilling equation (11). The other case follows analogously. With Definition 4 we get for $t \in[0, T]$ that

$$
\begin{aligned}
& v_{00}\left(t ;\left(1-R L_{12}^{\pi_{(1,1)}}(t)\right) X^{\pi_{(1,1)}}(t-)\right) \\
= & v_{00}\left(t ;\left(1-\pi_{(1,1)}(t)^{T} z^{(1)}\right)\left(1-\bar{\pi}_{(0,1)}(t)^{T} z^{(2)}\right) X^{\pi_{(1,1)}(t-)}\right) .
\end{aligned}
$$

Now we use the same idea as in the proof of Proposition 5. To prove the martingale property we use Itô's rule, the representation of $v_{00}(t ; x)$ from Proposition 2, Eq. (11) and get

$$
d v_{00}(t ; x(t))=v_{00}(t ; x(t))(1-\gamma) \pi_{(1,1)}(t)^{T} \sigma d W(t),
$$

for $x(t)=\left(1-\pi_{(1,1)}(t)^{T} z^{(1)}\right)\left(1-\bar{\pi}_{(0,1)}(t)^{T} z^{(2)}\right) X^{\pi_{(1,1)}}(t-)$. The solution of this SDE is given by

$$
\begin{array}{r}
v_{00}(t ; x(t))=v_{00}(0 ; x(0)) \cdot \exp \left((1-\gamma) \int_{0}^{t} \pi_{(1,1)}(s)^{T} \sigma d W(s)\right. \\
\left.-\frac{1}{2}(1-\gamma)^{2} \int_{0}^{t} \pi_{(1,1)}(s)^{T} \sigma \sigma^{T} \pi_{(1,1)}(s) d s\right),
\end{array}
$$

where the second factor is a martingale by Novikov's condition and the integrability assumptions of an admissible portfolio process. Together with the terminal condition $\pi(T)^{T} z^{(1)}=0$, we get the sub-scenario indifference property.

The following Corollary specifies the conditions under which a portfolio process that fulfills at least one of the two ODEs above is minimum constant.

Corollary 1 (Minimum constant sub-scenario indifference strategy) Let $\pi \in \mathbb{A}(x)$ be an admissible portfolio process and $t_{1}, t_{2} \in[0, T]$ with $t_{1} \leq t_{2}$. Further, let $\pi_{(1,1)}$ fulfill one of the following properties:

(i) $\pi_{(1,1)}$ is a solution of ODE (11) on $\left[t_{1}, t_{2}\right]$ and for all $t \in\left[t_{1}, t_{2}\right]$ it holds $R L^{\pi_{(1,1)}}(t)=$ $R L_{12}^{\pi(1,1)}(t)$

(ii) $\pi_{(1,1)}$ is a solution of $O D E(12)$ on $\left[t_{1}, t_{2}\right]$ and for all $t \in\left[t_{1}, t_{2}\right]$ it holds $R L^{\pi_{(1,1)}}(t)=$ $R L_{21}^{\pi(1,1)}(t)$.

Then we have $\mathbb{E}\left(M^{\pi}\left(\tau_{1}\right)\right)=\mathbb{E}\left(M^{\pi}\left(\tau_{2}\right)\right)$ for all $\left[t_{1}, t_{2}\right]$-valued stopping times $\tau_{1}$ and $\tau_{2}$.

Proof In case of property (i) the relative worst-case loss of $\pi$ is $R L^{\pi_{(1,1)}}(t)=R L_{12}^{\pi_{(1,1)}}(t)$ and with the definition of the minimum process $M^{\pi}$ it follows that

$$
\begin{aligned}
M^{\pi}(t) & =v_{00}\left(t ;\left(1-R L^{\pi_{(1,1)}}(t)\right) X^{\pi_{(1,1)}}(t-)\right) \\
& =v_{00}\left(t ;\left(1-R L_{12}^{\pi_{(1,1)}}(t)\right) X^{\pi_{(1,1)}}(t-)\right) \text { for } t \in\left[t_{1}, t_{2}\right] .
\end{aligned}
$$

By construction, $\pi$ is a sub-scenario indifference strategy for the sub-scenario $\xi_{1}<\xi_{2}$ on $\left[t_{1}, t_{2}\right]$, i.e. with the same arguments as in the proof of Proposition 8, we get that $v_{00}\left(t ;\left(1-R L_{12}^{\pi}(t)\right) X^{\pi}(t-)\right)$ is a martingale on $\left[t_{1}, t_{2}\right]$. The result follows with Doob's optional sampling theorem. For property (ii) the statement is proven analogously. 
Verification theorem for the multi-stress worst-case problem. The above considerations on sub-scenario indifference strategies allow us to formulate the verification theorem. Before we start, however, we would like to look at a short example, which illustrates the general procedure in a simplified way.

Example 1 We consider the most basic setting of two independent stocks, two possible stresses each affecting only one stock. Therefore we have $z^{(1)}=(k, 0)^{T}, z^{(2)}=(0, k)^{T}$ for $k \geq 0$ and $\sigma_{12}=\sigma_{21}=0$. With the notation $\bar{\pi}_{(1,0)}=\left(\bar{\pi}_{(1,0), 1}, \bar{\pi}_{(1,0), 2}\right)^{T}$ and $\bar{\pi}_{(0,1)}=\left(\bar{\pi}_{(0,1), 1}, \bar{\pi}_{(0,1), 2}\right)^{T}$ one can show that

$$
\begin{aligned}
\bar{\pi}_{(1,0), 1}^{\prime}(t) k= & \left(1-\bar{\pi}_{(1,0), 1}(t) k\right)\left(\bar{\pi}_{(1,0), 1}(t)\left(b_{1}-r\right)\right. \\
& \left.-\frac{1}{2} \gamma\left(\bar{\pi}_{(1,0), 1}(t)^{2} \sigma_{11}^{2}+\left(\pi_{1}^{*}\right)^{2} \sigma_{11}^{2}\right)\right), \\
\bar{\pi}_{(0,1), 2}^{\prime}(t) k= & \left(1-\bar{\pi}_{(0,1), 2}(t) k\right)\left(\bar{\pi}_{(0,1), 2}(t)\left(b_{2}-r\right)\right. \\
& \left.-\frac{1}{2} \gamma\left(\bar{\pi}_{(0,1), 2}(t)^{2} \sigma_{22}^{2}+\left(\pi_{2}^{*}\right)^{2} \sigma_{22}^{2}\right)\right),
\end{aligned}
$$

with $\bar{\pi}_{(1,0)}(T)^{T} z^{(1)}=\bar{\pi}_{(0,1)}(T)^{T} z^{(2)}=0$ and

$$
\bar{\pi}_{(1,0), 2}(t)=\pi_{2}^{*} \text { and } \bar{\pi}_{(0,1), 1}(t)=\pi_{1}^{*} \text { for } t \in[0, T] .
$$

Using Corollary 1 , it is easy to verify that $\bar{\pi}$ with $\bar{\pi}_{(1,1)}=\left(\bar{\pi}_{(1,0), 1}, \bar{\pi}_{(0,1), 2}\right)$ is a minimum constant strategy as it solves the ODEs (11) and (12). Due to the terminal conditions and the martingale properties we get, for all $t \in[0, T]$,

$$
v_{00}\left(t ;\left(1-R L_{12}^{\bar{\pi}_{(1,1)}}(t)\right) X^{\bar{\pi}_{(1,1)}}(t-)\right)=v_{00}\left(t ;\left(1-R L_{21}^{\bar{\pi}_{(1,1)}}(t)\right) X^{\bar{\pi}_{(1,1)}}(t-)\right),
$$

and we also have $R L^{\bar{\pi}_{(1,1)}}(t)=R L_{12}^{\bar{\pi}_{(1,1)}}(t)=R L_{21}^{\bar{\pi}_{(1,1)}}(t), t \in[0, T]$. To show that $\bar{\pi}$ solves the worst-case portfolio problem under stresses, we know, by the proof of Theorem 2, that it is enough to show that $\bar{\pi}$ solves the constrained portfolio optimization problem (10). We maximize

$$
\phi_{\gamma}(\pi)=r+\pi^{T}(b-r \underline{1})-\frac{1}{2} \gamma \pi^{T} \sigma \sigma^{T} \pi
$$

point wise in $\pi$. The maximizer in the unconstrained case is $\pi^{*}=\left(\pi_{1}^{*}, \pi_{2}^{*}\right)$ and by Lemma (1) we have

$$
\pi_{(1,0), 1}(t)<\pi_{1}^{*} \text { and } \pi_{(0,1), 2}(t)<\pi_{2}^{*} .
$$

Furthermore, the set of all strategies respecting the indifference frontier is

$$
\begin{aligned}
& I D(\bar{\pi})=\left\{\pi \in \mathbb{A}(x) \mid R L^{\pi_{(1,1)}}(t) \leq R L^{\bar{\pi}_{(1,1)}}(t) \text { for all } t \in[0, T]\right\} \\
= & \left\{\pi \in \mathbb{A}(x) \mid \pi_{(1,1)}(t)^{T} z^{(1)} \leq \bar{\pi}_{(1,1)}(t)^{T} z^{(1)} \wedge \pi_{(1,1)}(t)^{T} z^{(2)} \leq \bar{\pi}_{(1,1)}(t)^{T} z^{(2)}\right. \\
& \quad \text { for all } t \in[0, T]\} \\
= & \left\{\pi \in \mathbb{A}(x) \mid \pi_{(1,1), 1}(t) \leq \bar{\pi}_{(1,1), 1}(t) \wedge \pi_{(1,1), 2}(t) \leq \bar{\pi}_{(1,1), 2}(t) \text { for all } t \in[0, T]\right\},
\end{aligned}
$$

where we use again the notation $\pi_{(1,1)}=\left(\pi_{(1,1), 1}, \pi_{(1,1), 2}\right)$ for $\pi \in \mathbb{A}(x)$. Together with the quadratic form of (13) and the inequalities (14) we get the optimality of $\bar{\pi}$ in the constrained optimization problem (10) and thus the optimality in the worst-case portfolio problem under stresses. 
We are now able to determine a verification theorem.

Theorem 3 (Verification Theorem) Consider the worst-case portfolio optimization problem (5) with stress scenarios $\left(z^{(1)}, \xi_{1}\right)$ and $\left(z^{(2)}, \xi_{2}\right)$. Let $\bar{\pi} \in \mathbb{A}(x)$ be an admissible portfolio process and $\pi^{*}$ be the optimal portfolio process in the post stress problem, defined in Proposition 2. Assume that there exists a $k \geq 0$ and $0=t_{0}<t_{1}<\ldots<t_{k}<t_{k+1}=T$, such that on every interval $\left(t_{s}, t_{s+1}\right]$, with $s=0, \ldots, k$, the portfolio process $\bar{\pi}_{(1,1)}$ fulfills one of the following properties:

(i) For a $j \in\{1,2\}$, the portfolio process $\bar{\pi}_{(1,1)}(t)$ is given by

$$
\bar{\pi}_{(1,1)}(t)=\pi^{*}(t)-\frac{\lambda_{j}}{\gamma}\left(\sigma \sigma^{T}\right)^{-1} z^{(j)}
$$

where $\lambda_{j}$ is given by Eq. (18) below, with $\lambda_{j} \geq 0$, and $N_{j}(t)$ is the unique solution of ODE (19) with terminal condition $N_{j}\left(t_{s+1}\right)=\bar{\pi}_{(1,1)}\left(t_{s+1}\right)^{T} z^{(j)}$. Further, we have for all $t \in\left(t_{s}, t_{s+1}\right]$, that $R L^{\bar{\pi}_{(1,1)}}(t)=R L_{12}^{\bar{\pi}_{(1,1)}}(t)$ holds if $j=1$ and $R L^{\bar{\pi}_{(1,1)}}(t)=$ $R L_{21}^{\bar{\pi}_{(1,1)}}(t)$ if $j=2$.

(ii) The portfolio process $\bar{\pi}_{(1,1)}(t)$ is given by

$$
\bar{\pi}_{(1,1)}(t)=\pi^{*}(t)-\frac{\lambda}{\gamma}\left(\sigma \sigma^{T}\right)^{-1} z^{(1)}-\frac{\mu}{\gamma}\left(\sigma \sigma^{T}\right)^{-1} z^{(2)},
$$

where $\lambda$ and $\mu$ are given by Eqs. (20) and (21), with $\lambda \geq 0$ and $\mu \geq 0$, and $N(t)$ and $M(t)$ are the unique solutions of the ODEs (22) and (23) with terminal conditions $N\left(t_{s+1}\right)=\bar{\pi}_{(1,1)}\left(t_{s+1}\right)^{T} z^{(1)}$ and $M\left(t_{s+1}\right)=\bar{\pi}_{(1,1)}\left(t_{s+1}\right)^{T} z^{(2)}$. Furthermore, we have $R L_{12}^{\bar{\pi}_{(1,1)}}(t)=R L_{21}^{\bar{\pi}_{(1,1)}}(t)$ for all $t \in\left(t_{s}, t_{s+1}\right]$.

In case of property (ii), we require that $z^{(1)}$ and $z^{(2)}$ are linearly independent. Finally, we assume that $R L^{\bar{\pi}_{(1,1)}}(T)=0$. Then $\bar{\pi}$ solves the worst-case portfolio optimization problem (5) with stress scenarios $\left(z^{(1)}, \xi_{1}\right)$ and $\left(z^{(2)}, \xi_{2}\right)$.

Proof Let $\bar{\pi} \in \mathbb{A}(x)$ be an admissible portfolio process that fulfills the properties of the verification theorem. By Remark 3 it is enough to prove that $\bar{\pi}_{(1,1)}$ is optimal if both stresses can still occur. We use the idea of the proof of Theorem 2, especially part (ii). According to this, we need to show, that

- $\bar{\pi}$ is a minimum constant portfolio process, and

- $\bar{\pi}$ is optimal in the stress-free world under all processes respecting its indifference frontier, i.e. $\bar{\pi}$ solves the constrained optimization problem

$$
\max _{\pi \in I D(\bar{\pi})} \phi_{\gamma}\left(\pi_{(1,1)}(t)\right) \text { for all } t \in[0, T] .
$$

We look at the interval $\left(t_{s}, t_{s+1}\right]$ for $s \in\{0, \ldots, k\}$, and prove that these two conditions are fulfilled.

(i) By using Lagrangian-multiplier methods one can show that $\bar{\pi}$ with

$$
\bar{\pi}_{(1,1)}(t)=\pi^{*}(t)-\frac{\lambda_{j}}{\gamma}\left(\sigma \sigma^{T}\right)^{-1} z^{(j)}
$$

where

$$
\frac{\lambda_{j}}{\gamma}=\frac{\pi^{*}(t)^{T} z^{(j)}-N_{j}(t)}{\left(z^{(j)}\right)^{T}\left(\sigma \sigma^{T}\right)^{-1} z^{(j)}}
$$


and $N_{j}$ is the unique solution of

$$
\begin{aligned}
& N_{j}^{\prime}(t)=\left(1-N_{j}(t)\right)\left(f^{\left.\bar{\pi}_{(1,1)}(t)-\frac{\bar{\pi}_{(0,1)}^{\prime}(t)^{T} z^{(2)}}{1-\bar{\pi}_{(0,1)}(t)^{T} z^{(2)}}\right),} \quad \text { if } j=1,\right. \\
& N_{j}^{\prime}(t)=\left(1-N_{j}(t)\right)\left(f^{\left.\bar{\pi}_{(1,1)}(t)-\frac{\bar{\pi}_{(1,0)}^{\prime}(t)^{T} z^{(1)}}{1-\bar{\pi}_{(1,0)}(t)^{T} z^{(1)}}\right),} \quad \text { if } j=2,\right.
\end{aligned}
$$

with $N_{j}\left(t_{s+1}\right)=\bar{\pi}_{(1,1)}\left(t_{s+1}\right)^{T} z^{(j)}$ and

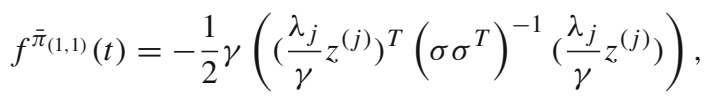

solves the following constrained optimization problem if $\lambda_{j} \geq 0$ :

$$
\max _{\pi \in \widetilde{I D}(\bar{\pi})} \phi_{\gamma}\left(\pi_{(1,1)}(t)\right) \text { for every } t \in\left(t_{s}, t_{s+1}\right] .
$$

Here, $\widetilde{I D}(\bar{\pi})$ is defined as

$$
\widetilde{I D}(\bar{\pi})=\left\{\pi \in \mathbb{A}(x) \mid \pi_{(1,1)}(t)^{T} z^{(j)} \leq \bar{\pi}_{(1,1)}(t)^{T} z^{(j)} \text { for } t \in\left(t_{s}, t_{s+1}\right]\right\} .
$$

Furthermore, $\bar{\pi}_{(1,1)}$ solves $\operatorname{ODE}(11)$ on $\left(t_{s}, t_{s+1}\right]$ if $j=1$ and ODE (12) if $j=2$. From $R L^{\bar{\pi}_{(1,1)}}(t)=R L_{12}^{\bar{\pi}_{(1,1)}}(t)$, respectively $R L^{\bar{\pi}_{(1,1)}}(t)=R L_{21}^{\bar{\pi}_{(1,1)}}(t)$, we get, that

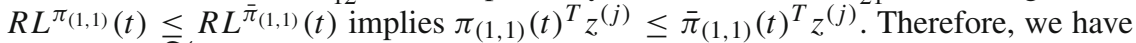
$I D(\bar{\pi}) \subseteq \widetilde{\widetilde{I D}}(\bar{\pi})$ and thus $\bar{\pi}$ solves the constrained optimization problem (17) on $\left(t_{s}, t_{s+1}\right]$. Further, $\bar{\pi}$ fulfills property (i) or (ii) of Corollary 1 on $\left(t_{s}, t_{s+1}\right]$.

(ii) Consider $\bar{\pi}$ with

$$
\bar{\pi}_{(1,1)}(t)=\pi^{*}(t)-\frac{\lambda}{\gamma}\left(\sigma \sigma^{T}\right)^{-1} z^{(1)}-\frac{\mu}{\gamma}\left(\sigma \sigma^{T}\right)^{-1} z^{(2)},
$$

where

$$
\begin{aligned}
& \frac{\lambda}{\gamma}=\left(\frac{\pi^{*}(t)^{T} z^{(1)}-N(t)}{\left(z^{(1)}\right)^{T}\left(\sigma \sigma^{T}\right)^{-1} z^{(1)}}-\frac{\left(\pi^{*}(t)^{T} z^{(2)}-M(t)\right)\left(z^{(1)}\right)^{T}\left(\sigma \sigma^{T}\right)^{-1} z^{(2)}}{\left(z^{(1)}\right)^{T}\left(\sigma \sigma^{T}\right)^{-1} z^{(1)}\left(z^{(2)}\right)^{T}\left(\sigma \sigma^{T}\right)^{-1} z^{(2)}}\right) / l, \\
& \frac{\mu}{\gamma}=\left(\frac{\pi^{*}(t)^{T} z^{(2)}-M(t)}{\left(z^{(2)}\right)^{T}\left(\sigma \sigma^{T}\right)^{-1} z^{(2)}}-\frac{\left(\pi^{*}(t)^{T} z^{(1)}-N(t)\right)\left(z^{(1)}\right)^{T}\left(\sigma \sigma^{T}\right)^{-1} z^{(2)}}{\left(z^{(1)}\right)^{T}\left(\sigma \sigma^{T}\right)^{-1} z^{(1)}\left(z^{(2)}\right)^{T}\left(\sigma \sigma^{T}\right)^{-1} z^{(2)}}\right) / l,
\end{aligned}
$$

with

$$
l=1-\frac{\left(\left(z^{(1)}\right)^{T}\left(\sigma \sigma^{T}\right)^{-1} z^{(2)}\right)^{2}}{\left(z^{(1)}\right)^{T}\left(\sigma \sigma^{T}\right)^{-1} z^{(1)}\left(z^{(2)}\right)^{T}\left(\sigma \sigma^{T}\right)^{-1} z^{(2)}} .
$$

Using the Cholesky decomposition and the Cauchy-Schwarz inequality we get $l>0$ by the linear independence assumption. Let $N$ and $M$ denote the unique solutions of

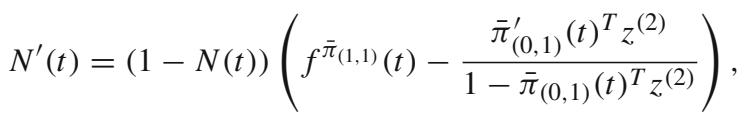




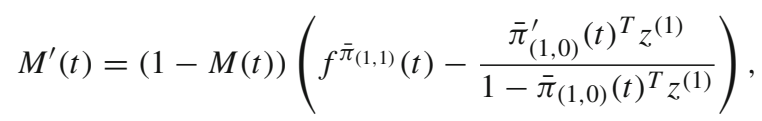

with $N\left(t_{s+1}\right)=\bar{\pi}_{(1,1)}\left(t_{s+1}\right)^{T} z^{(1)}$ and $M\left(t_{s+1}\right)=\bar{\pi}_{(1,1)}\left(t_{s+1}\right)^{T} z^{(2)}$ and

$$
f^{\bar{\pi}_{(1,1)}(t)}=-\frac{1}{2} \gamma\left(\left(\frac{\lambda}{\gamma} z^{(1)}+\frac{\mu}{\gamma} z^{(2)}\right)^{T}\left(\sigma \sigma^{T}\right)^{-1}\left(\frac{\lambda}{\gamma} z^{(1)}+\frac{\mu}{\gamma} z^{(2)}\right)\right) .
$$

Again, by using Lagrangian-multiplier methods and the Karush-Kuhn-Tucker conditions, one can show that $\bar{\pi}$ solves the following constrained optimization problem if $\lambda \geq 0$ and $\mu \geq 0$ :

$$
\max _{\pi \in \widetilde{I D}(\bar{\pi})} \phi_{\gamma}\left(\pi_{(1,1)}(t)\right) \text { for every } t \in\left(t_{s}, t_{s+1}\right],
$$

with

$$
\begin{aligned}
\widetilde{I D}(\bar{\pi})=\left\{\pi \in \mathbb{A}(x) \mid \pi_{(1,1)}(t)^{T} z^{(1)} \leq \bar{\pi}_{(1,1)}(t)^{T} z^{(1)}\right. \\
\\
\left.\wedge \pi_{(1,1)}(t)^{T} z^{(2)} \leq \bar{\pi}_{(1,1)}(t)^{T} z^{(2)} \text { for } t \in\left(t_{s}, t_{s+1}\right]\right\} .
\end{aligned}
$$

Furthermore, $\bar{\pi}_{(1,1)}$ solves ODE (11) and ODE (12). Using the definition of the relative worst-case loss and $R L_{12}^{\bar{\pi}_{(1,1)}}(t)=R L_{21}^{\bar{\pi}_{(1,1)}}(t)$ for all $t \in\left(t_{s}, t_{s+1}\right], R L^{\pi_{(1,1)}}(t) \leq$ $R L^{\bar{\pi}_{(1,1)}}(t)$ for $t \in\left(t_{s}, t_{s+1}\right]$ implies $\pi \in \widetilde{I D}(\bar{\pi})$ and therefore $I D(\bar{\pi}) \subseteq \widetilde{I D}(\bar{\pi})$. So $\bar{\pi}$ solves the constrained optimization problem (17) and fulfills property (i) and (ii) of Corollary 1 on $\left(t_{s}, t_{s+1}\right]$.

In summary, this means that $\bar{\pi}$ solves the constrained optimization problem and on each interval $\left(t_{s}, t_{s+1}\right]$ for $s=0, \ldots, k$ it fulfills at least one of the two properties of Corollary 1 . Thus, due to the right-continuity and the boundary condition $R L^{\bar{\pi}_{(1,1)}}(T)=$ $0, \bar{\pi}$ is minimum constant.

Remark 9 (Notes on the verification theorem) We make a few comments on the verification theorem.

1. If $\bar{\pi}_{(1,1)}$, for $\bar{\pi} \in \mathbb{A}(x)$, fulfills property (i) of the verification theorem on the whole interval $[0, T]$, then $\bar{\pi}$ is by construction a sub-scenario indifference strategy. An investor using this portfolio process prefers the stress order $\xi_{2}<\xi_{1}$ if $R L^{\bar{\pi}_{(1,1)}}(t)=R L_{12}^{\bar{\pi}_{(1,1)}}(t)$ and $\xi_{1}<\xi_{2}$ if $R L^{\bar{\pi}_{(1,1)}}(t)=R L_{21}^{\bar{\pi}_{(1,1)}}(t)$.

2. An investor with portfolio process $\bar{\pi} \in \mathbb{A}(x)$, where $\bar{\pi}_{(1,1)}$ fulfills property (ii) of the verification theorem on the interval $[0, T]$, with $\bar{\pi}_{(1,1)}(T)^{T} z^{(1)}=0$ and $\bar{\pi}_{(1,1)}(T)^{T} z^{(2)}=$ 0 , is indifferent concerning the time and order of occurrence of the two stresses, since then we have

$$
\begin{aligned}
& \mathbb{E}\left(v_{00}\left(\tau ;\left(1-R L_{12}^{\bar{\pi}_{(1,1)}}(\tau)\right) X^{\bar{\pi}_{(1,1)}}(\tau-)\right)\right) \\
= & \mathbb{E}\left(v_{00}\left(T ;\left(1-R L_{12}^{\bar{\pi}_{(1,1)}}(T)\right) X^{\bar{\pi}_{(1,1)}}(T-)\right)\right) \\
= & \mathbb{E}\left(v_{00}\left(T ;\left(1-R L_{21}^{\bar{\pi}_{(1,1)}}(T)\right) X^{\bar{\pi}_{(1,1)}}(T-)\right)\right) \\
= & \mathbb{E}\left(v_{00}\left(\tau ;\left(1-R L_{21}^{\bar{\pi}_{(1,1)}}(\tau)\right) X^{\bar{\pi}_{(1,1)}}(\tau-)\right)\right) \text { for all } \tau \in \Theta .
\end{aligned}
$$

3. The verification theorem in the log-utility case is obtained by setting $\gamma=1$. For this, note that we can compute sub-scenario indifference strategies in the log-utility case by using Itô's rule, analogous to the proof of Proposition 8. 


\section{Characterization of the solution and numerical illustration}

To apply the verification theorem we are now going to construct, in a heuristic way, general candidates for the optimal portfolio process. These heuristic constructions do not serve as an alternative to a proof of existence, but should make it easier to find a suitable process. As the numerical considerations show, the Lagrange multipliers appearing in the verification theorem depend to a large extent on the correlations of the stocks and the type of stresses. Due to the dependence on the ODEs, it must be checked numerically whether they fulfill the non-negativity conditions of the verification theorem. In the following we describe a procedure that helps to determine the optimal strategy.

In the numerical considerations of this section, the matrix $\sigma$ is a $d \times d$ lower triangular matrix of full rank.

Linearly dependent stresses. We consider the situation of two linearly dependent stresses $z^{(1)}=a z^{(2)}$, with $a \geq 1$. The proof of the verification theorem implies that a portfolio process which satisfies property (ii) solves the two ODEs (11) and (12). But this is only possible if the stresses are linearly independent. Hence, it is sufficient to consider the case of property (i). The linear dependency and the boundary condition $R L^{\bar{\pi}_{(1,1)}}(T)=0$ directly yield $\bar{\pi}_{(1,1)}(T)^{T} z^{(1)}=\bar{\pi}_{(1,1)}(T)^{T} z^{(2)}=0$. Here, where $z^{(1)}$ is the worse stress, it now makes sense to compute (15) for $j=1$, check the required properties and if necessary switch to $j=2$.

A simple example is shown in Fig. 1. Here, we have $r=0, \gamma=1.5, T=20$, one stock with $b=0.03, \sigma=0.2$, and two linearly dependent stresses $z^{(1)}=0.25$ and $z^{(2)}=0.15$. Solving the ODE (11) and verifying the conditions of part (i) of the verification theorem yield that the worst-case optimal strategy is the optimal sub-scenario indifference strategy for the sub-scenario $\xi_{1}<\xi_{2}$, i.e. one should be prepared for the larger stress occurring first. Linearly independent stresses. By assumption (2) and since $\bar{\pi}_{(1,0)}(T)^{T} z^{(1)}=0$ and $\bar{\pi}_{(0,1)}(T)^{T} z^{(2)}=0$, we know that

$$
\bar{\pi}_{(1,1)}(T) \in \arg \max \underset{\substack{\pi \in \mathbb{R}^{2} \quad \\ \pi^{T} z^{(1)} \leq 0 \\ \pi^{T} z^{(2)} \leq 0}}{ } \phi_{\gamma}(\pi)
$$

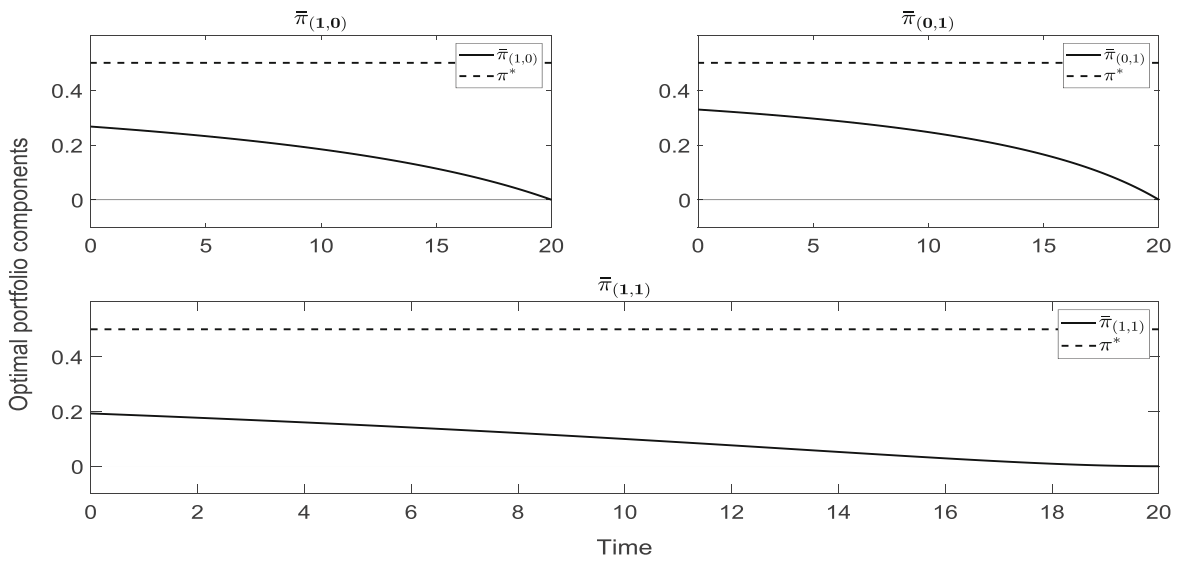

Fig. 1 One stock with $z^{(1)}=0.25$ and $z^{(2)}=0.15$ 


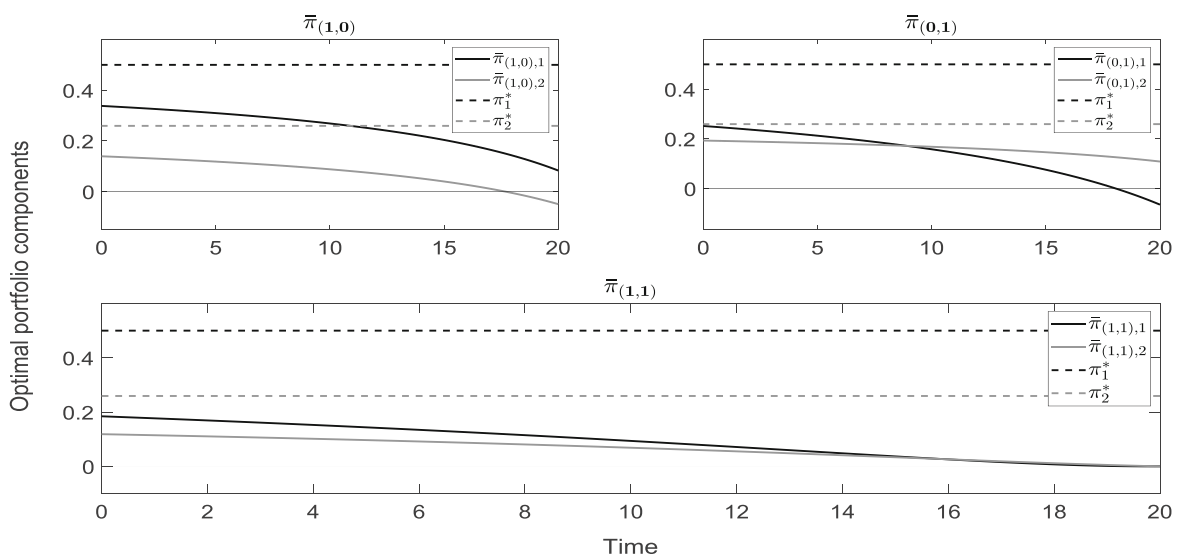

Fig. 2 Two uncorrelated stocks with $z^{(1)}=(0.15,0.25)^{T}$ and $z^{(2)}=(0.25,0.15)^{T}$

solves the boundary condition $R L^{\bar{\pi}_{(1,1)}}(T)=0$, since at least one of the constraints in the above optimization problem is fulfilled with equality for the optimal solution. If both constraints are fulfilled with equality we know that $R L^{\bar{\pi}_{(1,1)}}(T)=R L_{12}^{\bar{\pi}_{(1,1)}}(T)=R L_{21}^{\bar{\pi}_{(1,1)}}(T)$ and therefore we should start to construct $\bar{\pi}_{(1,1)}$ accordingly to (16). If, without loss of generality, $\bar{\pi}_{(1,1)}(T)^{T} z^{(1)}<0$ we know that $\bar{\pi}_{(1,1)}(T)^{T} z^{(2)}=0$ (otherwise $\pi^{*}$ would fulfill the constraints). Then, we construct $\bar{\pi}_{(1,1)}$ accordingly to (15) with $j=2$, since $R L^{\bar{\pi}_{(1,1)}}(T)=R L_{21}^{\bar{\pi}_{(1,1)}}(T)$.

Now we look at the interval $\left(t_{s}, t_{s+1}\right]$ for $s \in\{0, \ldots, k\}$ and a not yet defined $t_{s}$. If $\bar{\pi}_{(1,1)}$ is given by Eq. (15) with $j=1$ ( $j=2$ is similar) in $t_{s+1}$, we define $t_{s}$ as

$$
t_{s}=\sup \left\{t \leq t_{s+1} \mid R L^{\bar{\pi}_{(1,1)}}(t)>R L_{12}^{\bar{\pi}_{(1,1)}}(t)\right\} .
$$

In $t_{s}$ one should then check whether the transition to (16) is possible. On the other hand, if $\bar{\pi}_{(1,1)}$ is given by Eq. (16) in $t_{s+1}$ we define $t_{s}$ as

$$
t_{s}=\sup \left\{t \leq t_{s+1} \mid \lambda(t)<0 \vee \mu(t)<0\right\},
$$

where $\lambda$ and $\mu$ are interpreted as functions in $t$. Let us assume that w.l.o.g. $\lambda\left(t_{s}-\right) \geq 0$ and $\mu\left(t_{s}-\right)<0$. Then we should switch to (15) with $j=1$, since then, due to continuity, we expect $R L^{\bar{\pi}_{(1,1)}}\left(t_{s}-\right)=R L_{12}^{\bar{\pi}_{(1,1)}}\left(t_{s}-\right)$.

As an example, we consider two uncorrelated stocks with $r=0, \gamma=1.5, b=$ $(0.03,0.035), \sigma_{11}=0.2, \sigma_{22}=0.3$ and $T=20$. In Fig. 2 , we see the optimal portfolio process for $z^{(1)}=(0.15,0.25)^{T}$ and $z^{(2)}=(0.25,0.15)^{T}$. Here, $\bar{\pi}_{(1,1)}$ fulfills property (ii) of the verification theorem on the whole interval $[0, T]$. Hence, the investor is indifferent with respect to the order of occurrence of the stresses. That is not the case for the optimal portfolio process in Fig. 3. Here, we have $z^{(1)}=(0.25,0.25)^{T}$ and $z^{(2)}=(0.35,0.15)^{T}$. On the interval $[0,16.1]$ the process $\bar{\pi}_{(1,1)}$ fulfills property (ii) of the verification theorem and afterwards property (i) for $j=1$, i.e. from $t=16.1$ on, the investor prefers the occurrence of the stress $z^{(2)}$ first.

Further numerical illustrations. In the following, we give four further numerical examples to show the extent to which the choice of the stresses influences the construction of the optimal minimum constant portfolio process. In all examples, we use the same two negatively correlated stocks and step wise vary the stresses. We select the following parameters: $T=20$, 


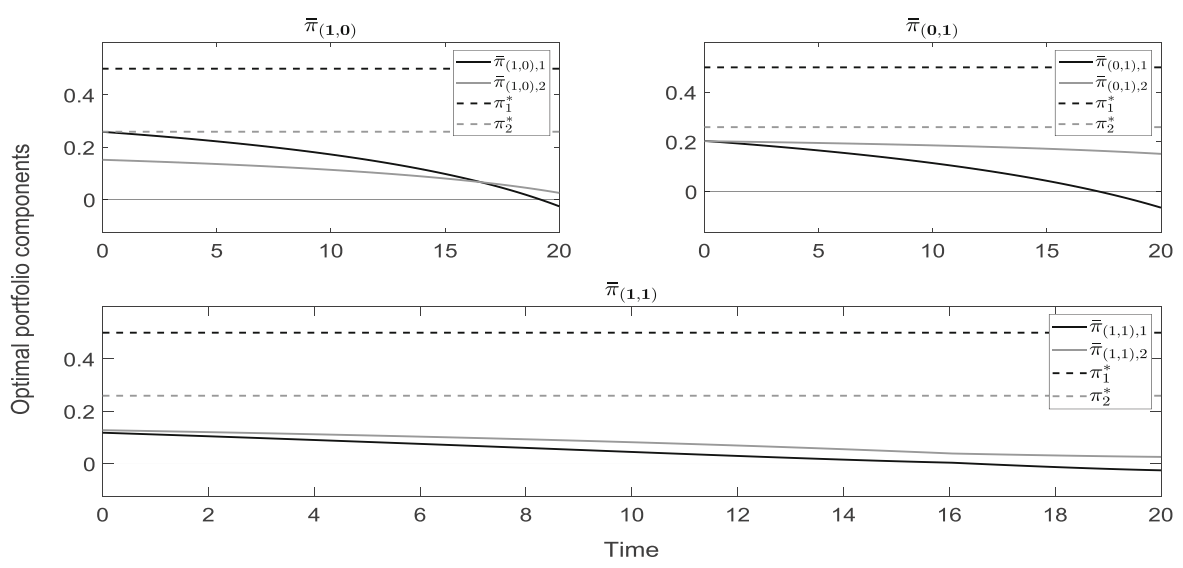

Fig. 3 Two uncorrelated stocks with $z^{(1)}=(0.25,0.25)^{T}$ and $z^{(2)}=(0.35,0.15)^{T}$

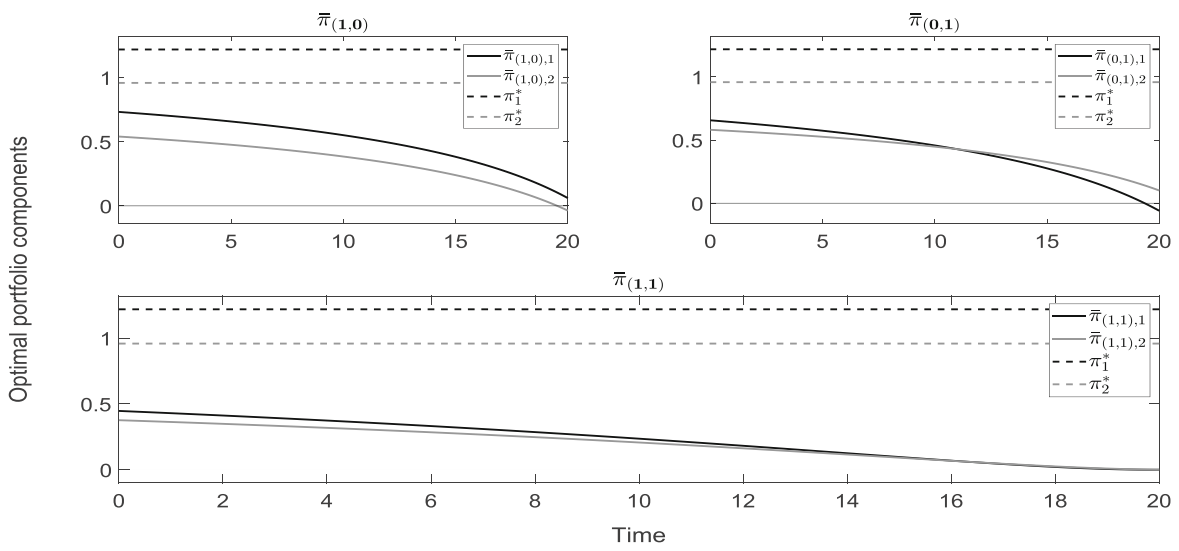

Fig. 4 Two negatively correlated stocks with $z^{(1)}=(0.15,0.25)^{T}$ and $z^{(2)}=(0.25,0.15)^{T}$

$\gamma=1.5, b=(0.03,0.035)$ and $\sigma_{11}=0.2, \sigma_{21}=-0.15, \sigma_{22}=0.2$. It follows that $\ln \left(S_{2}\right)$ has a volatility of 0.25 and the log-prices of the two stocks have a correlation of $\rho=-0.6$. In Fig. 4 we use the stresses $z^{(1)}=(0.15,0.25)^{T}$ and $z^{(2)}=(0.25,0.15)^{T}$. The symmetric stresses cause the optimal strategy to satisfy property (ii) of the verification theorem on the interval $[0, T]$ and the investor is therefore indifferent regarding time and order of occurrence of the stresses. Note that in relation to Fig. 2, the negative correlation leads to significantly higher investments in the risky assets.

In Fig. 5, we now slightly adjust stress $z^{(2)}$ and consider the stresses $z^{(1)}=(0.15,0.25)^{T}$ and $z^{(2)}=(0.15,0.15)^{T}$, so that $z^{(1)}$ can be regarded as the worse stress. The optimal strategy $\bar{\pi}_{(1,1)}$ fulfills property (i) for $j=1$ on the interval $[0,8.8]$ and afterwards property (ii) of the verification theorem. Hence, the investor first prefers the occurrence of stress $z^{(2)}$, since $R L^{\bar{\pi}_{(1,1)}}(t)=R L_{12}^{\bar{\pi}_{(1,1)}}(t)$, before being indifferent regarding the order of occurrence.

The time of transition from property (i) to (ii) is shifted back in time in Fig. 6 by further reducing stress $z^{(2)}$ and using the stresses $z^{(1)}=(0.15,0.25)^{T}$ and $z^{(2)}=(0.1,0.1)^{T}$. Here, $\bar{\pi}_{(1,1)}$ fulfills property (i) for $j=1$ on the interval $[0,15]$ and afterwards property (ii). 


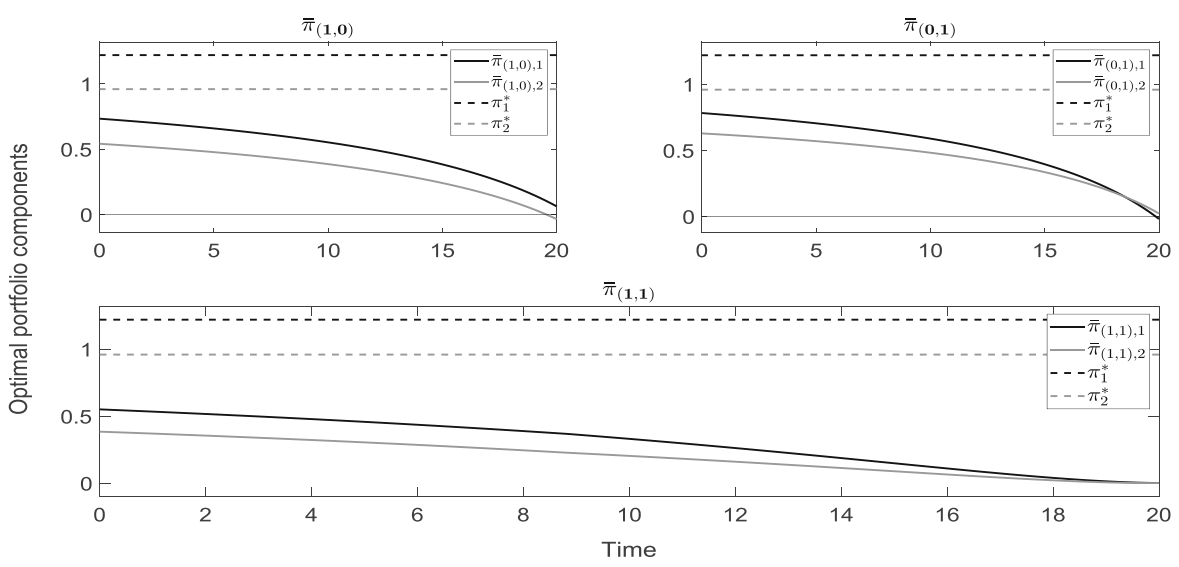

Fig. 5 Two negatively correlated stocks with $z^{(1)}=(0.15,0.25)^{T}$ and $z^{(2)}=(0.15,0.15)^{T}$

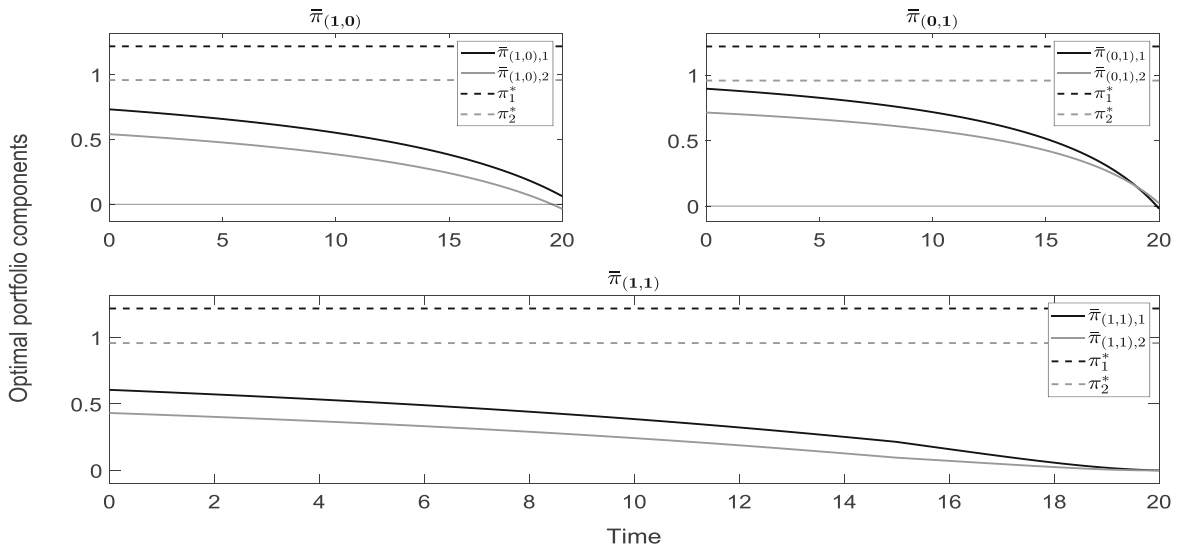

Fig. 6 Two negatively correlated stocks with $z^{(1)}=(0.15,0.25)^{T}$ and $z^{(2)}=(0.1,0.1)^{T}$

Finally, we swap the components of stress $z^{(1)}$ and consider the stresses $z^{(1)}=$ $(0.25,0.15)^{T}$ and $z^{(2)}=(0.1,0.1)^{T}$ in Fig. 7. Here we have two transitions between the properties of the verification theorem. On the interval $[0,14.4]$, the optimal strategy $\bar{\pi}_{(1,1)}$ satisfies property (i) for $j=1$, on the interval $[14.4,18.5]$ property (ii) and then again property (i), but this time for $j=2$. This in particular means that towards the time horizon the investor prefers stress $z^{(1)}$, which is actually perceived as worse. This is because there is a short position in the first stock $S_{1}$ if $\bar{\pi}_{(1,1)}$ fulfills property (i) for $j=2$ close to $T$.

\section{Conclusion}

We have modelled a portfolio optimization problem with possible stress scenarios by suitably adapting the worst-case scenario portfolio approach originally introduced in [13]. This included the introduction of different types of stresses and of an uncertain ordering of their occurrence. To deal with such a kind of problem, we generalized the concepts of the indiffer- 


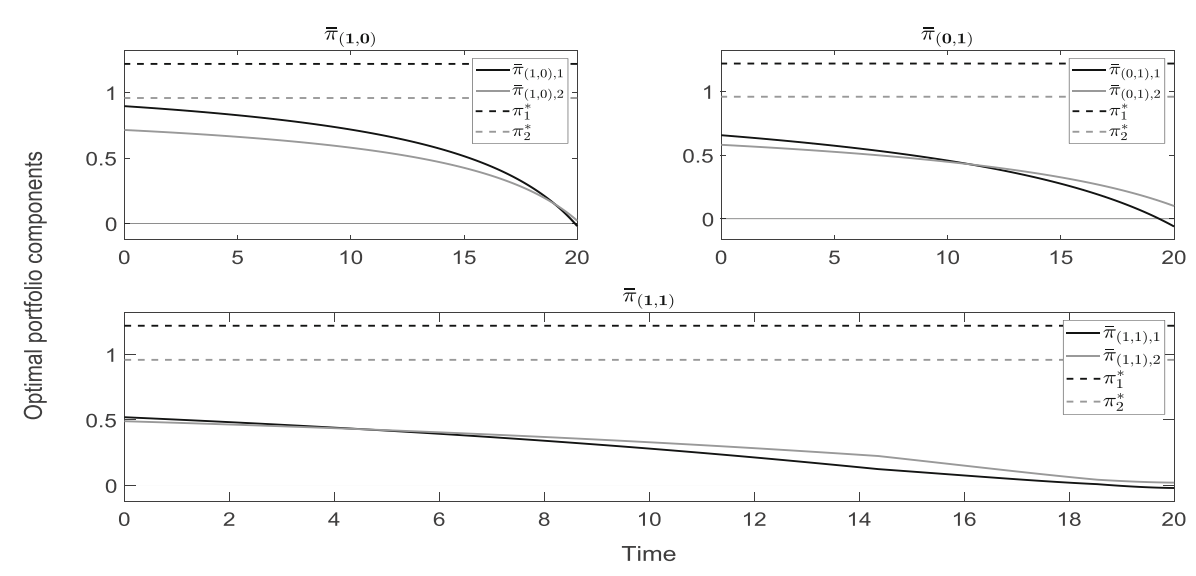

Fig. 7 Two negatively correlated stocks with $z^{(1)}=(0.25,0.15)^{T}$ and $z^{(2)}=(0.1,0.1)^{T}$

ence frontier and of the indifference-optimality principle as given in [20] via the introduction of the minimum constant process.

We succeed in proving the existence of a minimum constant process which solves the multiasset multi-stress worst-case problem in case that more than one stress can occur.

With the help of a verification theorem, we developed an algorithmic framework for coming up with candidates for the optimal worst-case portfolios and then verifying the guess by checking the validity of one of the two different sufficient conditions in the theorem.

Numerical examples highlighted various different effects. There can be settings where the investor globally is worst-case hedged against one particular ordering of the stresses. There can also be the situation where the investor is indifferent with respect to the ordering of the occurrence of the stresses, or where he/she is locally indifferent, but also locally is focused to concentrate on a particular ordering as the worst case.

Thus, the stress scenario setting is more complex than the classical worst-case portfolio problem. It is thus also not surprising that in the verification theorem we can only give sufficient conditions for a worst-case optimal portfolio strategy.

Acknowledgements We would like to thank two anonymous reviewers for careful reading, constructive comments and suggestions. Further, we thank the editor for constructive comments and suggestions.

Funding Open Access funding enabled and organized by Projekt DEAL.

Data availability Data sharing not applicable to this article as no datasets were generated or analysed during the current study.

Open Access This article is licensed under a Creative Commons Attribution 4.0 International License, which permits use, sharing, adaptation, distribution and reproduction in any medium or format, as long as you give appropriate credit to the original author(s) and the source, provide a link to the Creative Commons licence, and indicate if changes were made. The images or other third party material in this article are included in the article's Creative Commons licence, unless indicated otherwise in a credit line to the material. If material is not included in the article's Creative Commons licence and your intended use is not permitted by statutory regulation or exceeds the permitted use, you will need to obtain permission directly from the copyright holder. To view a copy of this licence, visit http://creativecommons.org/licenses/by/4.0/. 


\section{References}

1. Biagini, S., Pınar, M.Ç.: The robust merton problem of an ambiguity averse investor. Math. Financ. Econ. 11(1), 1-24 (2017)

2. Diez, F.: Yield Curves and Chance-Risk Classification: Modeling, Forecasting, and Pension Product Portfolios. Dissertation, TU Kaiserslautern (2020)

3. Commission, European: Commission delegated regulation (EU) 2015/35 of 10 October 2014 supplementing directive 2009/138/ec of the European parliament and of the council on the taking-up and pursuit of the business of insurance and reinsurance (Solvency II). Off. J. Eur. Union L12, 1-797 (2015)

4. European Insurance and Occupational Pensions Authority (EIOPA): Methodological Principles of Insurance Stress Testing. EIOPA-BoS-19/568 (2019)

5. Garlappi, L., Uppal, R., Wang, T.: Portfolio selection with parameter and model uncertainty: a multi-prior approach. Rev. Financ. Stud. 20(1), 41-81 (2006)

6. Insurance Regulation Committee of the IAA: Stress Testing and Scenario Analysis. https://www.actuaries. org/CTTEES_SOLV/Documents/StressTestingPaper.pdf (2013). Accessed 06 Apr 2021

7. Knight, F.H.: Risk, Uncertainty and Profit. Courier Corporation (2012)

8. Korn, R.: Worst-case scenario investment for insurers. Insur. Math. Econ. 36(1), 1-11 (2005)

9. Korn, R., Leoff, E.: Multi-asset worst-case optimal portfolios. Int. J. Theor. Appl. Finance 22(04), 1-24 (2019)

10. Korn, R., Menkens, O.: Worst-case scenario portfolio optimization: a new stochastic control approach. Math. Methods Oper. Res. 62(1), 123-140 (2005)

11. Korn, R., Seifried, F.T.: A worst-case approach to continuous-time portfolio optimization. Radon Ser. Comput. Appl. Math. 8, 327-345 (2009)

12. Korn, R., Steffensen, M.: On worst-case portfolio optimization. SIAM J. Control Optim. 46(6), 2013-2030 (2007)

13. Korn, R., Wilmott, P.: Optimal portfolios under the threat of a crash. Int. J. Theor. Appl. Finance 5(02), 171-187 (2002)

14. Lin, Q., Riedel, F.: Optimal consumption and portfolio choice with ambiguous interest rates and volatility. Econ. Theory 66, 6 (2020)

15. Merton, R.C.: Lifetime portfolio selection under uncertainty: the continuous-time case. Rev. Econ. Stat. 66, 247-257 (1969)

16. Neufeld, A., Nutz, M.: Robust utility maximization with Lévy processes. Math. Finance 28(1), 82-105 (2018)

17. Pflug, G., Wozabal, D.: Ambiguity in portfolio selection. Quant. Finance 7(4), 435-442 (2007)

18. Pliska, S.: A stochastic calculus model of continuous trading: optimal portfolios. Math. Oper. Res. 11, 371-382 (1986)

19. Schied, A.: Optimal investments for robust utility functionals in complete market models. Math. Oper. Res. 30, 750-764 (2005)

20. Seifried, F.T.: Optimal investment for worst-case crash scenarios: a martingale approach. Math. Oper. Res. 35(3), 559-579 (2010)

21. Talay, D., Zheng, Z.: Worst case model risk management. Finance Stoch. 6(4), 517-537 (2002)

Publisher's Note Springer Nature remains neutral with regard to jurisdictional claims in published maps and institutional affiliations. 